\title{
A MID-INFRARED CENSUS OF STAR FORMATION ACTIVITY IN BOLOCAM GALACTIC PLANE SURVEY SOURCES
}

\author{
Miranda K. Dunham ${ }^{1,2}$, Thomas P. Robitaille ${ }^{2,3,7}$, Neal J. Evans II $^{2}$, Wayne M. Schlingman ${ }^{4,8}$, \\ Claudia J. Cyganowski ${ }^{3,5,9}$, and James Urquhart ${ }^{6}$ \\ ${ }^{1}$ Department of Astronomy, Yale University, P.O. Box 208101, New Haven, CT 06520-8101, USA, miranda.dunham@yale.edu \\ ${ }^{2}$ Department of Astronomy, The University of Texas at Austin, 1 University Station C1400, Austin, Texas 78712-0259, USA \\ ${ }^{3}$ Harvard-Smithsonian Center for Astrophysics, 60 Garden Street, Cambridge, MA 02138, USA \\ ${ }^{4}$ Steward Observatory, University of Arizona, 933 North Cherry Ave., Tucson, AZ 85721, USA \\ ${ }^{5}$ Department of Astronomy, University of Wisconsin, Madison, WI 53706, USA \\ ${ }^{6}$ Australia Telescope National Facility, CSIRO Astronomy and Space Science, P.O. Box 76, Epping, NSW 1710, Australia \\ Received 2010 July 12; accepted 2011 February 4; published 2011 March 28
}

\begin{abstract}
We present the results of a search for mid-infrared signs of star formation activity in the $1.1 \mathrm{~mm}$ sources in the Bolocam Galactic Plane Survey (BGPS). We have correlated the BGPS catalog with available mid-IR Galactic plane catalogs based on the Spitzer Space Telescope GLIMPSE legacy survey and the Midcourse Space Experiment (MSX) Galactic plane survey. We find that 44\% (3712 of 8358) of the BGPS sources contain at least one mid-IR source, including 2457 of $5067(49 \%)$ within the area where all surveys overlap $\left(10^{\circ}<\ell<65^{\circ}\right)$. Accounting for chance alignments between the BGPS and mid-IR sources, we conservatively estimate that $20 \%$ of the BPGS sources within the area where all surveys overlap show signs of active star formation. We separate the BGPS sources into four groups based on their probability of star formation activity. Extended Green Objects and Red MSX Sources make up the highest probability group, while the lowest probability group is comprised of "starless" BGPS sources which were not matched to any mid-IR sources. The mean $1.1 \mathrm{~mm}$ flux of each group increases with increasing probability of active star formation. We also find that the "starless" BGPS sources are the most compact, while the sources with the highest probability of star formation activity are on average more extended with large skirts of emission. A subsample of 280 BGPS sources with known distances demonstrates that mass and mean $\mathrm{H}_{2}$ column density also increase with probability of star formation activity.
\end{abstract}

Key words: dust, extinction - infrared: stars - ISM: clouds - stars: formation

Online-only material: machine-readable table

\section{INTRODUCTION}

The formation of massive stars remains one of the prominent puzzles in astronomy (see review by McKee \& Ostriker 2007). A current goal of high-mass star formation studies is to determine an evolutionary sequence and lifetimes similar to the class system in low-mass star formation (e.g., Shu et al. 1987). While the evolutionary sequence is still open to considerable debate, one proposed sequence is summarized in recent reviews by Churchwell (2002) and Zinnecker \& Yorke (2007). This evolutionary sequence begins with prestellar cores or clumps: gravitationally bound overdensities within a molecular cloud which show signs of inward motion but have not yet begun to form a protostar. Since prestellar cores are lacking any internal heating, their temperatures are typically $10-20 \mathrm{~K}$ and their spectral energy distributions peak in the far-infrared.

Infrared dark clouds (IRDCs), seen in absorption against a diffuse mid-infrared background, are considered the cold precursors to star clusters (Rathborne et al. 2006), and thousands have been cataloged from the MSX and GLIMPSE surveys (Egan et al. 1998; Carey et al. 1998; Simon et al. 2006; Peretto \& Fuller 2009). While IRDCs may be the sites of the earliest stages of massive star formation, the clumps and cores within IRDCs can host massive young stellar objects (MYSOs) in various stages of evolution (e.g., Chambers et al. 2009; Rathborne et al. 2005,

\footnotetext{
7 Tinsley Visiting Scholar

8 Spitzer Postdoctoral Fellow

9 NSF Astronomy and Astrophysics Postdoctoral Fellow
}

2006, 2007, 2008, 2010). While a prestellar core is only seen as an IRDC if it is located at the near distance and there is enough background IR emission to absorb, IRDCs are seen in emission at far-infrared, submillimeter, and millimeter wavelengths, allowing for detection and identification of prestellar cores throughout the Galaxy.

The second stage in the proposed evolutionary sequence of Churchwell (2002) is hot cores: compact (diameter $<0.1 \mathrm{pc}$ ), dense $\left(n>10^{7} \mathrm{~cm}^{-3}\right)$, and warm $(T>100 \mathrm{~K})$ molecular cloud cores with high molecular line brightness temperatures (Kurtz et al. 2000), which are a result of internal heating from a massive protostar (Churchwell 2002). These hot cores will not contain any radio free-free emission because the infall rate will be high enough for the infalling material to absorb the UV flux from the central protostar.

Once the accretion begins to taper off, a small but detectable $\mathrm{H}$ II region will form. Thus, the third evolutionary stage is thought to include hyper-compact $\mathrm{H}$ II (HCH II) regions, which will rapidly expand to ultra-compact $\mathrm{H}_{\text {II }}$ (UCH II) regions. This stage can be characterized by the presence of radio emission (Churchwell 2002). The final stages are compact and classical $\mathrm{H}$ II regions. The $\mathrm{H}$ II region has now expanded and begun disrupting the parent molecular cloud while revealing the embedded stellar population in the optical and infrared.

This evolutionary sequence based on the size of $\mathrm{H}$ II regions may be too simplistic. Recent simulations of the gravitational collapse of a massive molecular cloud suggest that the size and morphology of $\mathrm{H}$ II regions can vary drastically during the formation of a massive star, with the $\mathrm{H}$ II region expanding and 
contracting repeatedly through the $\mathrm{HCH}$ II and $\mathrm{H}$ II classifications (Peters et al. 2010a, 2010b). These simulations suggest that the size of an $\mathrm{H}$ II region will only depend on age late in the lifetime of the $\mathrm{H}$ II region once accretion has halted.

Other evolutionary sequences have been proposed based on the presence of various types of masers, radio emission, thermal emission from warm dust, as well as any combination or complete lack of these star formation tracers (e.g., De Buizer et al. 2005; Minier et al. 2005; Ellingsen et al. 2007; Longmore et al. 2007; Purcell et al. 2009; Breen et al. 2010). These studies have included, at most, on the order of a hundred sources, and while detailed studies of small samples are crucial for understanding the results of large-scale surveys, Galaxy-wide samples are needed in order to solidify an evolutionary sequence.

Recent and ongoing (sub)millimeter wavelength continuum surveys of the Galactic plane are providing a novel inventory of star formation sites within the Galaxy at unprecedented sensitivity and resolution (BGPS, Aguirre et al. 2011; ATLASGAL, Schuller et al. 2009; JPS, Di Francesco 2008). These surveys are detecting thousands of sites of massive star formation and providing a statistically significant sample with which to begin studying star formation on a Galactic scale. However, these surveys alone cannot provide direct evidence of a source's evolutionary state and whether it is actively forming stars.

Fortunately, a wealth of other multiwavelength surveys that can provide evidence of a source's evolutionary state are already available or currently being observed. For example, the Spitzer Space Telescope GLIMPSE survey (Benjamin et al. 2003; Churchwell et al. 2009) at 3.6 to $8.0 \mu \mathrm{m}$ can provide information regarding the population of embedded young stellar objects (YSOs) found throughout the Galactic plane via mid-infrared colors (e.g., Robitaille et al. 2008) and the presence of outflows from massive young stellar objects traced by extended $4.5 \mu \mathrm{m}$ emission (e.g., Cyganowski et al. 2008). Similarly, the Red MSX Source survey (RMS; Hoare et al. 2004) has identified a reliable sample of massive YSOs by following up colorselected MSX YSO candidates. The Spitzer MIPS Galactic Plane Survey (MIPSGAL; Carey et al. 2009) has mapped the emission from warm dust in the Galactic plane at 24 and $70 \mu \mathrm{m}$ and can also identify embedded protostars. The ongoing Herschel Infrared Galactic Plane Survey (Hi-Gal; Molinari et al. 2010) will map the same region as the Spitzer surveys at 60-600 $\mu \mathrm{m}$ and will identify YSOs at wavelengths spanning the peaks of their spectral energy distributions. Together with the shorter wavelengths from Spitzer, Hi-Gal will provide accurate protostellar luminosities (e.g., Elia et al. 2010). At longer wavelengths, the Co-Ordinated Radio "N" Infrared Survey for High-mass Star Formation (CORNISH; Purcell et al. 2008) will provide a complete catalog of UCHII regions. To gain the most insight from the copious Galactic plane surveys, we must begin correlating their data sets.

In this paper, we present the results of a comparison of the Bolocam Galactic Plane Survey (BGPS) and available, Galaxywide mid-IR based YSO catalogs with the goal of characterizing the star formation activity within the BGPS sources. This work represents the first step in determining the evolutionary state of the BGPS sources. Further comparison to other Galactic plane surveys, such as MIPSGAL and CORNISH, is required and will be the subject of future papers. Section 2 presents the details of the various data sets included. The basic results are presented in Section 3, including the cross-matching method, a discussion of the prevalence of chance alignments, and the basic cross-matching statistics. Section 4 discusses trends in various millimeter properties as a function of star formation activity, and Section 5 provides a summary of the work.

\section{DATA SETS}

\subsection{The Bolocam 1.1 mm Galactic Plane Survey}

The Bolocam Galactic Plane Survey ${ }^{10}$ (BGPS; Aguirre et al. 2011) has surveyed $170 \mathrm{deg}^{2}$ of the Northern Galactic Plane in $1.1 \mathrm{~mm}$ continuum emission using the facility long-wavelength camera, Bolocam (Glenn et al. 2003; Haig et al. 2004), at the Caltech Submillimeter Observatory (CSO) on Mauna Kea. The survey consists of two distinct portions: a blind survey of the inner Galaxy spanning $-10.5<\ell<70.0$ where $|b|<0.5$ everywhere except for 1.0 sections in $\ell$ centered at $\ell=3^{\circ}, 15^{\circ}, 30^{\circ}$, and $31^{\circ}$ where $|b|<1.5$, and a targeted outer Galaxy portion including Cygnus-X $\left(70^{\circ}<\ell<90.5\right.$, $|b|<1.5)$, the Perseus Arm $\left(\ell \sim 111^{\circ}\right)$, IC1396 $\left(\ell \sim 99^{\circ}\right.$, $b \sim 3.5)$, the $\mathrm{W} 3 / 4 / 5$ region $\left(\ell \sim 135^{\circ}, b \sim 0.5\right)$, and the Gemini OB1 molecular cloud $\left(\ell \sim 190^{\circ}, b \sim 0.5\right)$.

Bolocam is a hexagonal array of 144 bolometers with a filter centered at $268 \mathrm{GHz}$ with an effective band center of $271.1 \mathrm{GHz}$ (Aguirre et al. 2011) and a bandwidth of $45 \mathrm{GHz}$. The filter excludes the $\mathrm{CO}(2-1)$ emission line. Each individual bolometer has a Gaussian beam with an FWHM of $31^{\prime \prime}$, and the instrument has an instantaneous field of view of 7'.5 due to the separation of the bolometers in the focal plane of the instrument. For more information regarding Bolocam, see Glenn et al. (2003) and Haig et al. (2004).

A reduction pipeline was created specifically for the BGPS survey, and is described in Aguirre et al. (2011). In order to retain the extended structure in the final maps, an iterative sky cleaning method was employed. At each iteration, a model of the sky emission is updated and subtracted from the overall data time stream to reveal a time stream containing only the astrophysical signal. The astrophysical signal is then mapped with $7^{\prime \prime} .2 \times 7^{\prime \prime} .2$ pixels and the effective beam size for the BGPS is $33^{\prime \prime}$.

Observations were obtained with scans along both $l$ and $b$ using the raster scan mode and a scan speed of $120^{\prime \prime} \mathrm{s}^{-1}$. Sensitivity to extended structure up to 5.9 angular size was retained by not utilizing chopping. The full flux density of objects larger than $5^{\prime} .9$ is not recovered, but long filaments with at least one dimension smaller than 5.9 are still detected (Aguirre et al. 2011). The flux density was calibrated based on observations of planets. Aguirre et al. (2011) compared BGPS with $1.2 \mathrm{~mm}$ data acquired with MAMBO on the IRAM $30 \mathrm{~m}$ (Rathborne et al. 2006; Motte et al. 2003, 2007) and SIMBA on the SEST $15 \mathrm{~m}$ (Matthews et al. 2009) and found that the v1.0 BGPS data were systematically lower and require a mean scaling factor of $1.5 \pm 0.15$ to align the BGPS fluxes with the previous MAMBO and SIMBA data sets. Here we present the v1.0 data with the scaling factor of $1.5 \pm 0.15$ applied.

Millimeter continuum sources were extracted from the iteratively mapped images using the BGPS source extraction software, Bolocat (Rosolowsky et al. 2010). Bolocat employs a seeded watershed method (Soille 1999) which first identifies all pixels above a signal-to-noise ratio $(\mathrm{S} / \mathrm{N})$ of 2.0 , identifies contiguous groups of pixels above this $\mathrm{S} / \mathrm{N}$, culls any groups which contain fewer than a beam's worth of pixels (22 7'.2 pixels), and then expands each remaining high signal-to-noise region through a nearest-neighbor algorithm to include all adjacent

\footnotetext{
10 See http://milkyway.colorado.edu/bgps/.
} 
pixels above an $\mathrm{S} / \mathrm{N}$ of 1.0. Extending the region to include pixels down to $1 \sigma$ is based on the reasoning that marginal emission next to regions of significant emission is likely real. The software then breaks each region up into smaller substructures based on the contrast between local maxima. Through the nearest-neighbor algorithm, each source is assigned a group of contiguous pixels and a source number. Bolocat then produces a label map, in which every pixel assigned to a source is assigned the source number. Source properties are then computed via emission-weighted moments. All flux-dependent properties presented in this work are based on the integrated flux density which is simply the sum of all pixels assigned to a BGPS source. This flux density avoids contamination from nearby sources that can occur with aperture photometry. For more details regarding source extraction and parameter estimation, see Rosolowsky et al. (2010).

In the generation of the Bolocam catalog (Rosolowsky et al. 2010) we evaluated both the completeness limit (false negatives as a function of $\mathrm{S} / \mathrm{N}$ ) and the spurious detection incidence (false positives as a function of catalog parameters). The completeness study was presented as part of the original catalog manuscript. The catalog parameters were specifically chosen to minimize false detections as well as false negatives. To evaluate the falsepositive incidence, we inserted an array of 2600 false sources into the data time stream for a blank field of observation. The time stream was for the $l=111$ field which had been cleaned of signal as a byproduct of the iterative mapping procedure (Aguirre et al. 2011). The injected sources had a range of brightnesses and the recovery fraction as a function of brightness established the completeness limit. The algorithm recovered 0 false detections - all sources in the final catalog were the injected sources. While this may be surprising, the catalog's relatively high effective completeness limit of $5 \sigma$ is a byproduct of eliminating false detections. Taking this as $0 \pm 1$ over the $4 \mathrm{deg}^{2}$ area of the field leads to an upper limit of $\sim 40$ false detections in the survey, or $0.5 \%$. We note that the injected sources were point sources and extended source recovery tests found that the BGPS catalog software often broke up extended emission into multiple objects, but it did not lead to the false detection of sources not associated with emission. Based on this test, we believe that the BGPS catalog contains only a very small number of false sources.

The BGPS catalog is $>99 \%$ complete at the $5 \sigma$ level. While $\sigma$ varies across the survey due to the varying number of observations and observing conditions, the survey as a whole is $>98 \%$ complete at the $0.4 \mathrm{Jy}$ level (see Figure 9 in Rosolowsky et al. 2010). Assuming a dust temperature of $20 \mathrm{~K}$ (Dunham et al. 2010), this completeness level translates into a minimum mass of $5 M_{\odot}, 21 M_{\odot}, 130 M_{\odot}, 524 M_{\odot}$, and $750 M_{\odot}$ at $1 \mathrm{kpc}, 2 \mathrm{kpc}, 5 \mathrm{kpc}, 10 \mathrm{kpc}$, and $12 \mathrm{kpc}$, respectively. However, since the BGPS filters out diffuse emission on scales larger than 5.9 (Aguirre et al. 2011), the types of sources the BGPS is sensitive to depends on distance (see Section 7.2 of Dunham et al. 2010 and Section 6 of Rosolowsky et al. 2010). At the nearest distances (up to approximately $2 \mathrm{kpc}$ ), the BGPS will be sensitive to cores (mass $M=0.5-5 M_{\odot}$, volume-averaged density $n=10^{4}-10^{5} \mathrm{~cm}^{-3}$, and physical radius $R=0.01-0.1 \mathrm{pc}$; Bergin \& Tafalla 2007), while at intermediate distances the BGPS will be sensitive to clumps $\left(M=50-500 M_{\odot}, n=10^{3}-10^{4} \mathrm{~cm}^{-3}, R=0.15-1.5 \mathrm{pc}\right.$; Bergin \& Tafalla 2007), and at the furthest distances BGPS will detect entire molecular clouds $\left(M=10^{3}-10^{4} M_{\odot}, n=50-500\right.$ $\mathrm{cm}^{-3}, R=1-7.5 \mathrm{pc}$; Bergin \& Tafalla 2007).
The BGPS images and catalog have been released to the public and are hosted by the Infrared Processing and Analysis Center via the NASA/IPAC Infrared Science Archive ${ }^{11}$ (IPAC).

\subsection{GLIMPSE Red Source Catalog}

In addition, we made use of the GLIMPSE survey, a Spitzer/IRAC Legacy survey of the Galactic mid-plane (Benjamin et al. 2003; Churchwell et al. 2009) at 3.6, 4.5, 5.8, and $8.0 \mu \mathrm{m}$. The GLIMPSE I survey covers $10^{\circ} \leqslant|\ell| \leqslant 65^{\circ}$ and $|b| \leqslant 1^{\circ}$ and the GLIMPSE II survey fills in the region for $|\ell|<10^{\circ}$ with $|b| \leqslant 1^{\circ}$ for $|\ell|>5^{\circ},|b| \leqslant 1.5$ for $2^{\circ}<|\ell| \leqslant 5^{\circ}$, and $|b| \leqslant 2^{\circ}$ for $|\ell| \leqslant 2^{\circ}$. The total area of the GLIMPSE I and II surveys is $274 \mathrm{deg}^{2}$, and the overlap region with the BGPS survey is $-10.5<\ell<65^{\circ},|b|<0.5$ and $|b|<1.0$ at $\ell=3^{\circ}, 15^{\circ}, 30^{\circ}$, and $31^{\circ}$. The data products include both highly reliable Catalogs, and less reliable but more complete archives.

Robitaille et al. (2008, hereafter R08) compiled a highly reliable census of 18,949 GLIMPSE point sources with excess emission at mid-infrared wavelengths, of which 11,649 were classified as candidate YSOs, and 7300 as candidate asymptotic giant branch (AGB) stars. The AGB classification was further separated into two categories consisting of "standard" AGB stars and "extreme" AGB stars. The "extreme" AGB stars have high mass-loss rates and therefore significant circumstellar dust. In this paper, we refer to the "standard" and "extreme" AGB stars as sAGB and xAGB stars, respectively, and both types together as simply AGB stars. The separation of the sources into YSOs and AGB stars was based on IRAC and MIPS color and magnitude selection criteria, and was only approximate. In reality, $50 \%-70 \%$ of the sources are likely to be YSOs, and $30 \%-50 \%$ are likely to be AGB stars.

The R08 census was designed to maximize reliability. As the sensitivity to point sources decreases in areas of bright diffuse emission-which are common at $8 \mu \mathrm{m}$ - the census used stringent magnitude selection criteria so as not to be affected by this. As a result, the R08 census may be missing fainter YSOs that are still detected by GLIMPSE. Therefore, for the current work, we compile a list of red GLIMPSE sources that were missed in the R08 census, with the caveat that this complementary list is therefore less reliable, and is background rather than flux limited, especially at $8 \mu \mathrm{m}$.

We constructed this complementary sample as follows. We first increased the reliability of the GLIMPSE catalog fluxes in a similar way to R08. First, each position in the GLIMPSE survey was observed at least twice, and we therefore removed fluxes from the catalog that depend only on a single detection. We required the fractional rms of the individual detections and the fractional error in the combined flux to be less than $15 \%$, otherwise the flux was removed. We then rejected sources that were not detected at least at 4.5 and $8.0 \mu \mathrm{m}$, and finally we selected the red sources by requiring [4.5] $-[8.0]>0.75$, a less stringent color selection than that used for R08 ([4.5] - [8.0] > 1.0). This results in 64,859 sources being selected, excluding the R08 sources, which we refer to as additional GLIMPSE sources.

Robitaille et al. (2008) estimated that the red source catalog is $65.7 \%$ complete. In addition, they explored the range of luminosities of YSOs detectable as a function of distance. The sensitivity of the GLIMPSE survey results in a lower flux limit, and Robitaille et al. (2008) imposed an upper flux limit in order to exclude point sources with unreliable fluxes due to saturation.

\footnotetext{
11 See http://irsa.ipac.caltech.edu/data/BOLOCAM_GPS/.
} 
They used the models of Robitaille et al. (2006) to calculate average [4.5] and [8.0] magnitudes for stage I and II YSOs as a function of luminosity, and calculated the range of distances at which a YSO would be included in their red source catalog. A $1 L_{\odot}$ YSO within $1 \mathrm{kpc}$ will be included in the red source catalog, while a $10 L_{\odot}$ YSO will be included if it is between 0.2 and $2 \mathrm{kpc}$. A $10^{3} L_{\odot}$ YSO between 2 and $10 \mathrm{kpc}$ will be included in the R08 catalog, and a $10^{4} L_{\odot}$ YSO between 4 and $11 \mathrm{kpc}$ will also be included. The most luminous YSOs will only be included at the farthest distances due to the upper flux limit imposed to avoid saturation, while the lowest luminosity sources will only be found at the nearest distances due to the sensitivity limits of the GLIMPSE survey (see Figure 19 and Section 4.5 of R08).

\subsection{Extended Green Objects Catalog}

The GLIMPSE catalogs and archives are point source catalogs, and therefore exclude any extended YSOs. In particular, massive embedded YSOs driving outflows can in some cases be seen as extended sources at $4.5 \mu \mathrm{m}$, as this band contains $\mathrm{H}_{2}$ emission lines as well as CO band heads (Reach et al. 2006; Smith et al. 2006). These lines can be excited by shocks, which are expected to be present where outflows interact with the interstellar medium. Cyganowski et al. (2008) compiled a list of 304 such objects, termed Extended Green Objects ${ }^{12}$ (EGOs), from the GLIMPSE I survey. The EGOs are likely tracing outflows from massive YSOs since they show a strong correlation with IRDCs and class II methanol masers (Cyganowski et al. 2009), both of which are associated with early stages of massive star formation (Cyganowski et al. 2008). The overlap area with the BGPS survey is $10^{\circ}<\ell<65^{\circ},|b|<0.5$, and $|b|<1^{\circ} .0$ at $\ell=$ $15^{\circ}, 30^{\circ}$, and $31^{\circ}$.

The EGO catalog is certainly not a complete census of YSOs with shocked outflows. The requirement of extended $4.5 \mu \mathrm{m}$ emission likely resulted in the preferential inclusion of YSOs at relatively small distances, as the angular extent decreases with distance. Also, as noted in Cyganowski et al. (2008), EGOs near bright sources or in areas with significant polycyclic aromatic hydrocarbon (PAH) emission and diffuse green emission extended on scales less than $10^{\prime \prime}$ are likely absent from the catalog.

Thus, the EGO catalog may include more massive, higher luminosity, nearby sources which were excluded from the R08 catalog because of the saturation limits.

\subsection{Red MSX Source Catalog}

The RMS survey ${ }^{13}$ (Hoare et al. 2004; Urquhart et al. 2008a) was designed to follow-up and confirm MYSO candidates selected from the Midcourse Space Experiment (MSX) Galactic plane survey (Price et al. 2001). The Galactic plane survey included all longitudes from $0^{\circ}$ to $360^{\circ}$, and latitudes within $|b|<5^{\circ}$ and consisted of imaging at 8.28, 12.13, 14.65, and $21.3 \mu \mathrm{m}$ with a resolution of $18^{\prime \prime}$ at all wavelengths (Price et al. 2001). A total of 1992 MYSO candidates were identified using color selection criteria. Low- and intermediate-mass YSOs were excluded from the catalog. All MYSO candidates were subsequently followed up to confirm their nature (Mottram et al. 2007; Urquhart et al. 2007a, 2007b, 2008b, 2009a, 2009b) and were classified into the following categories: "Evolved star," "PN,"

\footnotetext{
12 The adjective "green" comes from the fact that these objects appear as green in IRAC three color images, since these often use $4.5 \mu \mathrm{m}$ for the green channel.

13 See http://www.ast.leeds.ac.uk/RMS.
}

"OH/IR star," "Young/old star?," "H II regions," "H II/YSO," and "YSO." In this work we include the "PN," "OH/IR star," and "Evolved star" classifications in a general "Evolved star" category. The "YSO/H II" classification is assigned when both an $\mathrm{H}$ II region and YSO are present or if a definitive classification is not possible, while the "Young/old" classification is assigned to sources which look like evolved stars but have strong $\mathrm{CO}$ emission along their line of sight, resulting in an uncertain classification. The BGPS overlaps the RMS survey where $\ell \geqslant 10^{\circ}$.

Unlike the R08 sample, the RMS selection criteria did not include an upper limit on fluxes, and as such the highest luminosity sources at all distances will be included in the catalog. YSOs with $L \sim 10^{3} L_{\odot}$ will be included to $3 \mathrm{kpc}$, while $10^{4} L_{\odot}$ YSOs will be included to a distance of $10 \mathrm{kpc}$, and $10^{5} L_{\odot}$ YSOs will be included across the entire Galaxy (see Figure 2 of Urquhart et al. 2008a). The luminosity range of the RMS survey overlaps with the range in the R08 red source catalog, and fills in the region of luminosity space excluded from the R08 catalog because of the saturation limitations.

\section{RESULTS}

\subsection{Cross-matching Method}

The data sets were cross-matched based on spatial coincidence. All infrared sources located within a BGPS source as defined by the label maps (see Section 2.1) are considered to be associated with the BGPS source. The label maps produced as a part of the BGPS source extraction (Rosolowsky et al. 2010) represent the full extent of each $1.1 \mathrm{~mm}$ continuum emission source down to the $1 \sigma$ level of the original image. We require that the catalog position of each mid-IR source fall within a pixel assigned to a BGPS source. The EGO sources subtend more than a single BGPS pixel; however, we find that ignoring the extended emission and simply using the catalog position corresponding to the peak of the $4.5 \mu \mathrm{m}$ emission does not adversely affect the matching statistics for the EGO catalog. Figure 1 shows the label map and corresponding image centered near $l \sim 6^{\circ}$ with the positions of the matched (crosses and plus signs) and unmatched (diamonds and circles) R08 and additional GLIMPSE sources, respectively. The catalog parameters for each BGPS source (major and minor axes and position angle) are displayed as the black ellipses. The BGPS sources clearly do not fill the black ellipses, highlighting the benefit of using the label maps rather than the BGPS mean source properties when cross-matching catalogs.

\subsection{Chance Alignments}

In matching various catalogs of mid-IR sources to the BGPS sources, we need to take into account the possibility of chance alignment of mid-IR sources with BGPS clumps. Since we do not have distance estimates to the mid-IR sources or most BGPS sources, we cannot determine which mid-IR sources that lie along the same line of sight as a BGPS source are in fact chance alignments. Instead, we can estimate the fraction of chance alignments statistically.

In order to do this, we first consider the fraction of the BGPS area that contains millimeter emission and assume that the midIR sources are evenly distributed across the survey area. In this case, the fraction of area covered with millimeter emission corresponds to the fraction of mid-IR sources that are chance alignments with the millimeter continuum emission. We calculate this fraction for the region that is common to all surveys: $10^{\circ}<\ell<65^{\circ}$, and $|b| \leqslant 0.5$ with extensions to $|b| \leqslant 1.0$ as described in Section 2.1. We find that $2.1 \%$ of the BGPS area 


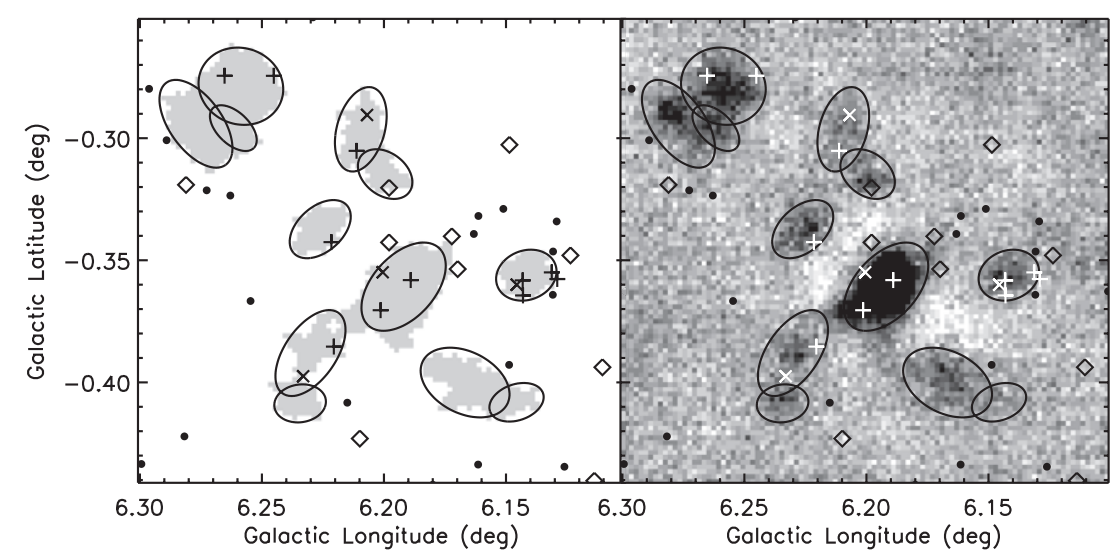

Figure 1. Left: the grayscale image shows the label map from a small portion of the BGPS. Right: the corresponding BGPS image shown in inverted grayscale ranging from 0.2 to $-0.1 \mathrm{Jy}_{\text {beam }}^{-1}$. In both images, the black ellipses mark the positions and sizes of the extracted BGPS sources as determined from emission-weighted moments. Crosses and diamonds represent GLIMPSE R08 red sources which are and are not associated with a BGPS source, respectively. Plus signs and circles mark additional GLIMPSE sources which were matched and not matched with a BGPS source, respectively. Note the BGPS source near $(\ell, b) \sim(6.23,-0.39)$. The ellipse encloses many pixels which do not include millimeter emission, thus demonstrating the benefit of using the label map for matching rather than the mean source properties which are shown here as the black ellipses.

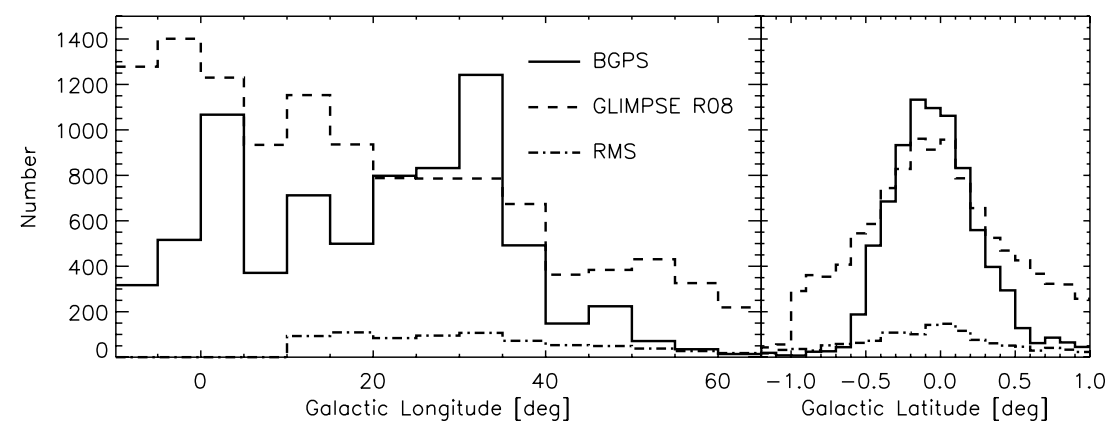

Figure 2. Distribution in Galactic longitude (left) and Galactic latitude (right) of the BGPS sources (solid line), GLIMPSE red sources with $\ell \geqslant-10^{\circ}$ (dashed), and all RMS sources (dash-dotted line). The EGO catalog is not plotted due to the small number of sources (84). The sources are clearly concentrated toward the Galactic mid-plane $\left(b \sim 0^{\circ}\right)$, and also show structure in Galactic longitude.

contains source emission, and therefore $2.1 \%$ of the mid-IR sources will overlap millimeter continuum emission by chance.

In reality, the distribution of the mid-IR sources is not even across the survey areas, but is concentrated towards the Galactic mid-plane, and also decreases with increasing longitude (Figure 2). Furthermore, even a population of mid-IR sources unrelated to star formation could display a similar nonuniform spatial distribution. To obtain a more realistic estimate of chance alignments, we attempt to preserve the large-scale distribution of the mid-IR sources by duplicating the catalogs and randomizing each source's coordinates within a circle of radius $1^{\circ}$ (in cases where mid-IR sources fell out of their original survey area, their positions were re-randomized). In this way, any population unrelated to star formation would preserve its large-scale distribution and would not show a difference in the number of chance alignments, while any star-forming population that was originally preferentially aligned with BGPS emission on arcsecond to arcminute scales would lose such a correlation. Figure 3 shows the distributions of Galactic longitude (left) and latitude (right) for the GLIMPSE red sources with $\ell \geqslant-10^{\circ}$ for the original (solid lines) and randomized (dashed lines) catalogs. Overall, the trends in the distributions of the original catalog are preserved in the randomized catalog although the peak in sources toward $b \sim 0^{\circ}$ is slightly decreased. To investigate the effect of the randomization radius used, we repeated the experiment with a radius of 0.1 and found that the fraction of chance alignments increases slightly. All GLIMPSE R08 source types (xAGB, sAGB, and YSO) taken together have $8.1 \%$ chance alignments with a $1^{\circ}$ randomization. If we use only a 0.1 randomization, we expect $10.7 \%$ chance alignments. The choice of radius for the randomization does not severely alter the chance alignment results.

Multiple randomized catalogs were created for each mid-IR data set in the interest of obtaining an error bar on the number of chance alignments. For the GLIMPSE catalogs, we randomized the catalogs 10 times, while for the EGO and RMS catalogs we randomized the catalogs 100 times to avoid issues due to small-number statistics. Column 5 of Table 1 presents the percentage of matched mid-IR source types that are expected to be chance alignments, and Column 9 shows the percentage of BGPS sources expected to be matched due to chance alignments with each mid-IR source type. Overall, around $7 \%$ of mid-IR sources would be expected to match the BGPS emission by chance alignments.

\subsection{Matching Statistics of the Mid-IR Sources}

Columns 2-5 of Table 1 present a summary of the matching statistics for each mid-IR catalog, including both the number and fraction of mid-IR sources that are matched to BGPS emission. The EGOs are most often matched to BGPS emission, with $90 \%$ of EGO sources matched (more than 10 times that expected from random chance alignment). In comparison, the 


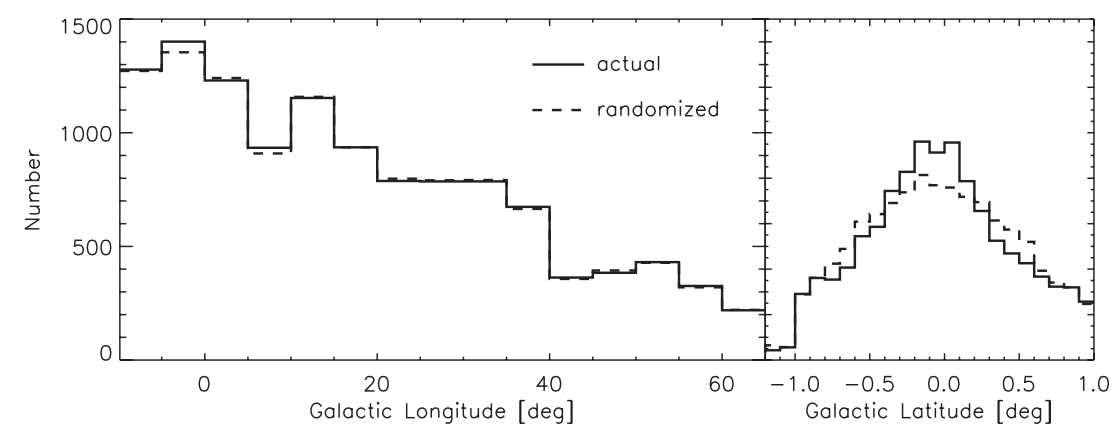

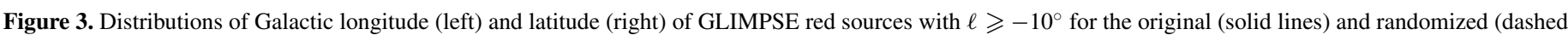

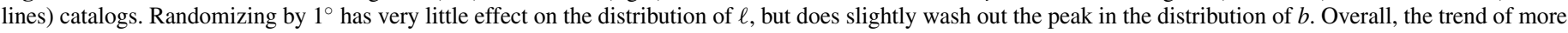
sources located near the Galactic mid-plane $\left(b \sim 0^{\circ}\right)$ is maintained.

Table 1

Matching Statistics

\begin{tabular}{|c|c|c|c|c|c|c|c|c|c|}
\hline \multirow[t]{2}{*}{ Catalog } & \multirow{2}{*}{$\begin{array}{c}\text { Objects in } \\
\text { Overlap Region }\end{array}$} & \multicolumn{3}{|c|}{ Objects Matching BGPS Sources } & \multirow{2}{*}{$\begin{array}{c}\text { BGPS in } \\
\text { Overlap Region }\end{array}$} & \multicolumn{3}{|c|}{ BGPS Sources Containing Objects } & \multirow[t]{2}{*}{ Confidence } \\
\hline & & Number & Percent & Randomized \% & & Number & Percent & Randomized \% & \\
\hline \multicolumn{10}{|l|}{ GLIMPSE R08 } \\
\hline YSOs & 5053 & 1692 & 33.5 & $8.2 \pm 0.4$ & 7360 & 1273 & 17.3 & $5.1 \pm 0.3$ & 70.5 \\
\hline sAGB & 1540 & 169 & 11.0 & $7.2 \pm 0.8$ & 7360 & 167 & 2.3 & $1.4 \pm 0.2$ & 36.8 \\
\hline xAGB & 1746 & 325 & 18.6 & $8.8 \pm 0.5$ & 7360 & 298 & 4.0 & $1.9 \pm 0.1$ & 52.1 \\
\hline GLIMPSE additional & 27278 & 5788 & 21.2 & $7.9 \pm 0.2$ & 7360 & 2929 & 39.8 & $21.3 \pm 0.5$ & 46.3 \\
\hline EGOs & 84 & 79 & 90.0 & $8.3 \pm 4.0$ & 5067 & 71 & 1.4 & $0.13 \pm 0.05$ & 90.8 \\
\hline RMS total & 608 & 414 & 68.1 & $8.4 \pm 1.2$ & 6062 & 374 & 6.2 & $0.78 \pm 0.11$ & 88.6 \\
\hline Unclassified/other & 26 & 13 & 50.0 & $7.2 \pm 4.7$ & 6062 & 13 & 0.21 & $0.029 \pm 0.019$ & 86.5 \\
\hline Evolved star & 133 & 12 & 9.0 & $6.8 \pm 2.2$ & 6062 & 12 & 0.20 & $0.14 \pm 0.047$ & 26.8 \\
\hline Young/old star? & 22 & 16 & 72.7 & $8.8 \pm 6.2$ & 6062 & 16 & 0.26 & $0.029 \pm 0.020$ & 89.0 \\
\hline H II region & 211 & 180 & 85.3 & $10.4 \pm 2.2$ & 6062 & 168 & 2.8 & $0.33 \pm 0.067$ & 88.1 \\
\hline H II/YSO & 91 & 85 & 93.4 & $8.8 \pm 3.1$ & 6062 & 85 & 1.4 & $0.12 \pm 0.041$ & 91.6 \\
\hline \multirow[t]{2}{*}{ YSO } & 124 & 108 & 87.1 & $6.9 \pm 2.3$ & 6062 & 106 & 1.7 & $0.13 \pm 0.044$ & 92.5 \\
\hline & & & & & Total & 3712 & 44.4 & & \\
\hline
\end{tabular}

RMS sources have a lower overall matched fraction (68\%). However, if we consider the different types of RMS sources separately, it becomes apparent that the types of RMS sources associated with star formation (e.g., H II regions, H II/YSO, and YSO) have a much higher fraction of matches with BGPS sources. Table 1 lists the matching statistics for the RMS sources by category. The young/old star, $\mathrm{H}$ II region, $\mathrm{H}$ II/YSO, and YSO categories have matched fractions approximately $8-10$ times the fraction expected by chance alignments $(80 \%-90 \%)$. In contrast, the fraction of RMS evolved stars matched with BGPS emission is perfectly consistent with that expected from chance alignments. Of the 133 RMS sources classified as AGB stars within the "Evolved stars" category, only 12 were matched with BGPS sources.

The GLIMPSE R08 YSOs tend to be less often associated with millimeter emission (33\%, or four times that expected from chance alignments). This low fraction could be due to several reasons. First, stage II YSOs, which are present in the GLIMPSE R08 catalog, no longer have an envelope and are unlikely to possess the density and angular size required for a detection in the BGPS (Dunham et al. 2010, Section 7.2). Secondly, the separation of YSOs and AGB stars in the GLIMPSE red source catalog was only approximate, and refining the magnitude and color selection criteria could result in a higher fraction of matched YSOs. The fraction of AGB stars from the GLIMPSE R08 catalog matched to millimeter emission is slightly above the level expected for chance alignments. This could be due to the approximate YSO/AGB separation which results in contamination from genuine YSOs in the sources classi- fied as AGB stars, or it could be indicative of some AGB sources being present in the BGPS catalog. Indeed, the dust around some AGB and post-AGB stars is detected at millimeter wavelengths (e.g., Buemi et al. 2007; Dehaes et al. 2007; Ladjal et al. 2010) and it is possible that some BGPS sources are actually evolved stars. The RMS team has performed followup observations and has identified the sources in their catalog which are AGB stars. Although the number of RMS "Evolved stars" matched with a BGPS source is consistent with the number expected due to chance alignments, we can use the matching statistics to estimate the number of GLIMPSE R08 AGB stars expected to be matched with a BGPS source. If we assume that the matching statistics for the RMS AGB stars within the "Evolved stars" category (12 of 133 were matched with a BGPS source, 9.0\%) also apply to the R08 AGB stars, then we expect 296 R08 AGB stars to be matched with BGPS sources. We find that a total of 494 R08 AGB stars are matched with BGPS sources, 198 more than expected based on the matching statistics of the RMS AGB stars. If the RMS statistics are representative of the R08 AGB stars as well, then roughly $60 \%$ of the matched R08 AGB stars are likely to be AGB stars and $40 \%$ are likely to be YSOs. Further observations are required to determine the nature of the AGB sources which are matched with BGPS sources. A future paper will further explore the matching statistics in order to assess the validity of the magnitude and color cuts made to define the YSO GLIMPSE red source list presented in R08.

Finally, the additional GLIMPSE source catalog contains $21 \%$ of sources that are matched with millimeter emission (almost three times the level expected from chance alignments), 
Table 2

Mid-IR Content of the BGPS Sources

\begin{tabular}{|c|c|c|c|c|c|c|c|c|c|}
\hline \multirow[t]{2}{*}{ Source $^{\mathrm{a}}$} & \multirow{2}{*}{$\begin{array}{l}\text { R.A. }^{\text {b }} \\
(\mathrm{J} 2000)\end{array}$} & \multirow{2}{*}{$\begin{array}{l}\text { Decl. }^{b} \\
(\mathrm{~J} 2000)\end{array}$} & \multirow{2}{*}{$\begin{array}{l}\ell^{\mathrm{b}} \\
\left({ }^{\circ}\right) \\
\end{array}$} & \multirow{2}{*}{$\begin{array}{l}b^{\mathrm{b}} \\
\left({ }^{\circ}\right) \\
\end{array}$} & \multirow{2}{*}{$\begin{array}{c}\text { Radius }^{\mathrm{c}} \\
\left({ }^{\prime \prime}\right) \\
\end{array}$} & \multicolumn{4}{|c|}{ Number Matched } \\
\hline & & & & & & EGO & $\mathrm{RMS}^{\mathrm{d}}$ & R08 & GLIM \\
\hline G000.000+00.057 & 266.34562 & -28.89994 & 0.00383 & 0.06324 & 38.4 & 0 & 0 & 0 & 1 \\
\hline G000.004+00.277 & 266.13967 & -28.78620 & 0.00663 & 0.27649 & 79.6 & 0 & 0 & 0 & 3 \\
\hline G000.006-00.135 & 266.55075 & -28.99171 & 0.01896 & -0.13780 & 82.1 & 0 & 0 & 0 & 3 \\
\hline G000.010+00.157 & 266.26410 & -28.83834 & 0.01913 & 0.15628 & 81.4 & 0 & 0 & 7 & 3 \\
\hline G000.016-00.017 & 266.42551 & -28.93289 & 0.01216 & -0.01361 & 93.2 & 0 & 0 & 0 & 6 \\
\hline G000.018-00.431 & 266.83618 & -29.14625 & 0.01632 & -0.43125 & $<16.5$ & 0 & 0 & 0 & 2 \\
\hline G000.020+00.033 & 266.38130 & -28.90146 & 0.01881 & 0.03579 & 77.2 & 0 & 0 & 0 & 7 \\
\hline G000.020-00.051 & 266.46830 & -28.94505 & 0.02127 & -0.05191 & 62.3 & 0 & 0 & 1 & 1 \\
\hline G000.022+00.251 & 266.17683 & -28.78469 & 0.02494 & 0.24951 & $<16.5$ & 0 & 0 & 1 & 1 \\
\hline G000.034-00.437 & 266.85490 & -29.13193 & 0.03704 & -0.43782 & $<16.5$ & 0 & 0 & 0 & 0 \\
\hline
\end{tabular}

Notes.

a The BGPS source name is based on the Galactic coordinates of the peak of the $1.1 \mathrm{~mm}$ emission.

$\mathrm{b}$ The coordinates of the geometric centroid of the $1.1 \mathrm{~mm}$ emission.

c For sources unresolved compared to the beam, the radius is set to an upper limit equal to half the beam size, 16 .' 5 .

d Only young RMS types ("H II region," "H II/YSO," "YSO," and "Young/old star?") are included here.

(This table is available in its entirety in a machine-readable form in the online journal. A portion is shown here for guidance regarding its form and content.)

indicating that it does contain a fraction of genuinely young stars.

\subsection{Star Formation Activity in BGPS Sources}

In Columns 6-9 of Table 1, we present the detailed numbers / fractions of BGPS sources containing each type of mid-IR source. We assume that the following mid-IR source types indicate star formation within the matched BGPS source: GLIMPSE R08 YSOs, sAGB, and xAGB stars; GLIMPSE additional sources; EGOs; young RMS source types including "Young/old star," "H II region," "H II/YSO," and "YSO." As previously mentioned (Section 3.3), we use the R08 AGB catalogs as an indicator of star formation because of the approximate separation of the YSOs and AGB sources and the resulting contamination of the R08 AGB catalogs by YSOs (see Section 2.2). Further observations would be required to determine if R08 AGB sources matched with BGPS sources are YSOs or AGB stars, and we note that some AGB stars matched with BGPS sources will actually be AGB stars.

A total of 3712 BGPS sources were identified as containing signs of star formation via the alignment of a mid-IR source, with the caveat that some of the matched R08 AGB stars are actually AGB stars and not indicative of star formation. This equates to $44 \%$ of the entire BGPS catalog, although this is a lower limit to the true fraction since the mid-IR catalogs do not overlap completely with the BGPS catalog. Only the RMS catalog overlaps the BGPS at $l>65^{\circ}$, and unlike the GLIMPSE survey it will only identify the brightest mid-IR sources. If we consider only the region where all surveys overlap $\left(10^{\circ}<l<65^{\circ}\right)$, we find a total of 2457 BPGS sources containing a mid-IR source out of 5067 BGPS sources in the inner Galaxy (48\%).

In order to calculate the true number and fraction of BGPS sources that show signs of active star formation, we must account for the occurrence of chance alignments. As previously discussed, the number of BGPS sources likely to have chance alignments were calculated for each mid-IR source catalog (the product of Columns 6 and 9 in Table 1). We simply subtract the number of BGPS sources identified as chance alignments from the number of BGPS sources which were matched with each individual mid-IR source type, and find that 1472 of the 8358 $(17.6 \%)$ BGPS sources in the entire catalog are reliable matches.
If we restrict this calculation to the 5067 BGPS sources in the area where all surveys overlap $\left(10^{\circ} \leqslant \ell \leqslant 65^{\circ}\right)$, we estimate that $1035(20.4 \%)$ BGPS sources are reliably matched with a mid-IR source. These percentages are conservative estimates of the percentage of BGPS sources that are actively forming stars and are driven so low due to the large number of BGPS sources expected to be identified via chance alignments with the additional GLIMPSE sources (1573 for the entire catalog and 976 for the overlap area only).

Since we are unable to identify which mid-IR sources are chance alignments with our current data set, we consider all matches in the subsequent discussion. Table 2 presents the number of mid-IR sources within each BGPS source. Column 1 gives the BGPS source name, Columns 2 and 3 give the R.A. and decl. of the geometric centroid, while Columns 4 and 5 give the Galactic coordinates. The angular radius of each BGPS source is given in Column 6. The number of matched sources from the EGO, RMS, GLIMPSE R08, and additional GLIMPSE catalogs are given in Columns 7-10, respectively. The full table is included in the online journal only.

We define a confidence value for each type which is the percent of matched BGPS sources which are not due to chance alignments. This is defined as the number of BGPS sources with a matching mid-IR source minus the number expected from chance alignments, divided by the number of BGPS sources with a matching mid-IR source, and is given in Column 10 of Table 1.

This confidence value allows us to estimate the probability that a match with a particular mid-IR source indicates ongoing star formation in the BGPS source. The calculated confidence values range from $26 \%$, for BGPS sources matched with RMS evolved stars, to roughly $90 \%$, for the BGPS sources matched to EGO and young RMS sources. The confidence is around 70\% for the GLIMPSE R08 YSOs, around 50\% for the additional GLIMPSE sources, and around $50 \%$ and $40 \%$ for the GLIMPSE R08 xAGB and sAGB stars, respectively.

\section{DISCUSSION}

In this section, we consider only the 5067 BGPS sources located in the region where all catalogs overlap $\left(10^{\circ} \leqslant \ell \leqslant 65^{\circ}\right)$. In order to compare the properties of the BGPS sources as a 


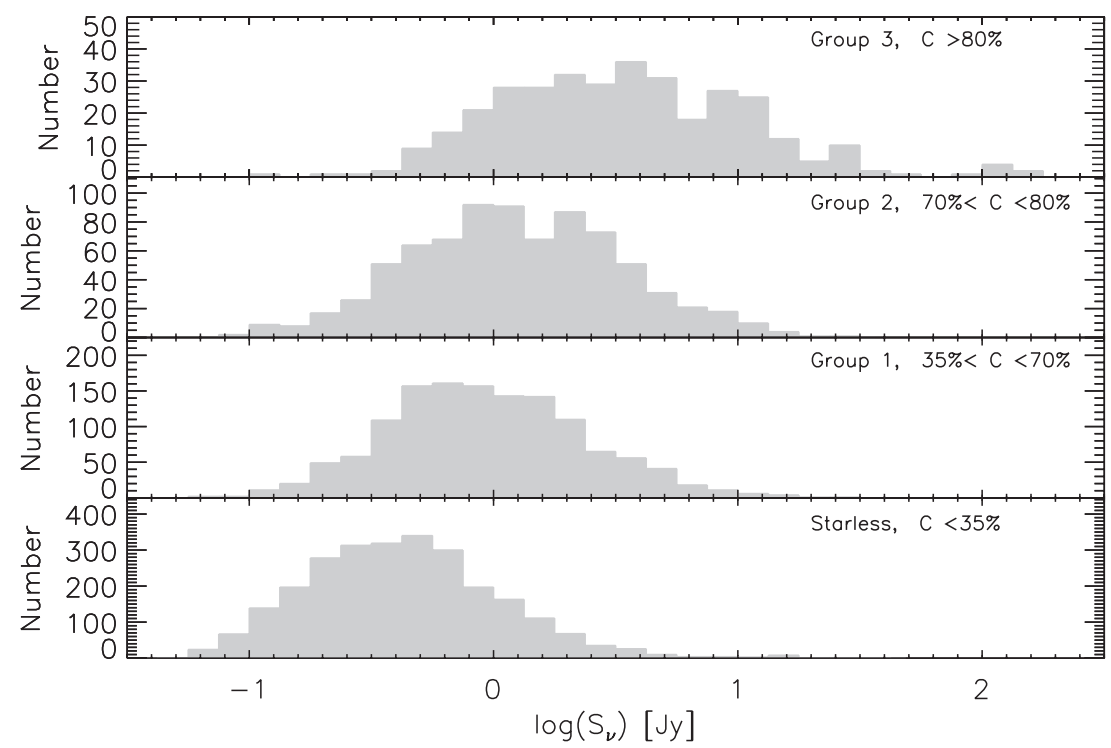

Figure 4. Number of sources as a function of the BGPS source flux for the different probability groups of BGPS sources. Only the 5067 BGPS sources in the region where all catalogs overlap are included. Note the vertical scale is different for each probability group. The range of confidence values for each probability group is given in the upper-right corner of each panel.

function of the star formation activity they contain, we define a classification scheme based on the confidence parameter listed in Table 1. We separate the BGPS sources into four groups representing the probability of star formation activity.

3. BGPS sources containing at least one EGO or young RMS (Young/old star, H II region, H II/YSO, or YSO) source (corresponding to a confidence of $>80 \%$ ).

2. BGPS sources containing no EGO or young RMS source, but at least one GLIMPSE R08 YSO (corresponding to a confidence of $70 \%$ ).

1. BGPS sources containing no EGO, young RMS source, or GLIMPSE R08 YSO, but at least one GLIMPSE R08 AGB star or additional GLIMPSE source (corresponding to a confidence of $40 \%-50 \%$ ). The inclusion of the R08 sources classified as AGB stars may seem counterintuitive, but they are contaminated by genuine YSOs and are therefore to some extent a star formation indicator.

0 . The remaining BGPS sources, which show no sign of star formation in the above catalogs. We refer to these sources as "starless." These may in fact be forming stars that are too faint to be detected, or too young and too deeply embedded to be visible in the mid-IR.

In this classification scheme, a BGPS source is assigned to a group based on the highest confidence mid-IR source type it was matched with. For example, a BGPS source matched with an EGO, R08 YSO, and five additional GLIMPSE sources would be assigned to group 3 based on the presence of the EGO source, while a BGPS source without the EGO, but with an R08 YSO and five additional GLIMPSE sources would be assigned to group 2 based on the presence of the R08 YSO.

Probability groups 0-3 are composed of 2610, 1324, 793, and 340 BGPS sources, respectively. The trend of fewer BGPS sources with increasing confidence parameter reflects the smaller number of mid-IR sources in the catalogs required for each higher confidence category. We note that the actual probability of active star formation for each BGPS source is unknown. The probability we refer to throughout this paper is a measure of the probability that the mid-IR source is a real association rather than a chance alignment.

\subsection{Observed Properties}

Figure 4 shows the number of BGPS sources as a function of the flux density integrated over the entire source for the BGPS sources in each probability group. BGPS sources in group 3 with the highest probability of star formation activity have the highest mean flux, $8.0 \mathrm{Jy}$, with a standard deviation about the mean of $18.0 \mathrm{Jy}$. The mean flux decreases with probability of star formation activity, with a mean of $0.71 \mathrm{Jy}$ and standard deviation of $1.4 \mathrm{Jy}$ for the "starless" sources. The flux distributions are asymmetric with tails extending to large fluxes. For example, the distribution of "starless" sources has a high flux tail extending to approximately $30 \mathrm{Jy}$.

The $1.1 \mathrm{~mm}$ BGPS flux densities could be contaminated by free-free emission from ionized gas if the BGPS source is coincident with an $\mathrm{H}$ II region or planetary nebula, for example. The trend of increasing flux density with probability of star formation could be due to an increase in the contamination from free-free with increasing probability of star formation. To quantify the free-free contamination, we have compared the BGPS flux densities with integrated intensities from $6 \mathrm{~cm}$ radio continuum observations toward RMS sources (Urquhart et al. 2009a). We have identified 223 radio continuum sources from Urquhart et al., which coincide with 159 unique BGPS sources. In cases where multiple radio continuum sources coincide with a BGPS source, we have simply taken the sum of the individual $6 \mathrm{~cm}$ fluxes for comparison to the $1.1 \mathrm{~mm}$ flux. Assuming that the $6 \mathrm{~cm}$ emission is on the optically thin side of the free-free spectrum, we scale the $6 \mathrm{~cm}$ emission to $1.1 \mathrm{~mm}$ via $S_{v}(\mathrm{ff}, 1.1 \mathrm{~mm})=S_{v}(6 \mathrm{~cm})(1.1 \mathrm{~mm} / 60 \mathrm{~mm})^{0.1}$ since $S_{\nu}$ (free-free) $\propto v^{-0.1}$. If the $6 \mathrm{~cm}$ emission is on the optically thick side of the free-free spectrum, then $S_{v}(\mathrm{ff}, 1.1 \mathrm{~mm})$ underestimates the free-free flux density, as the spectrum increases as $v^{2}$ on the optically thick side (Mezger \& Henderson 1967; Tielens 2005).

Figure 5 shows the distribution of the ratio of the free-free flux density at $1.1 \mathrm{~mm}$ to the $1.1 \mathrm{~mm}$ BGPS flux density. The dotted line marks the mean fractional contribution from free-free of $0.039 \pm 0.066$, and the dashed line marks the median of 0.013. The minimum and maximum free-free 


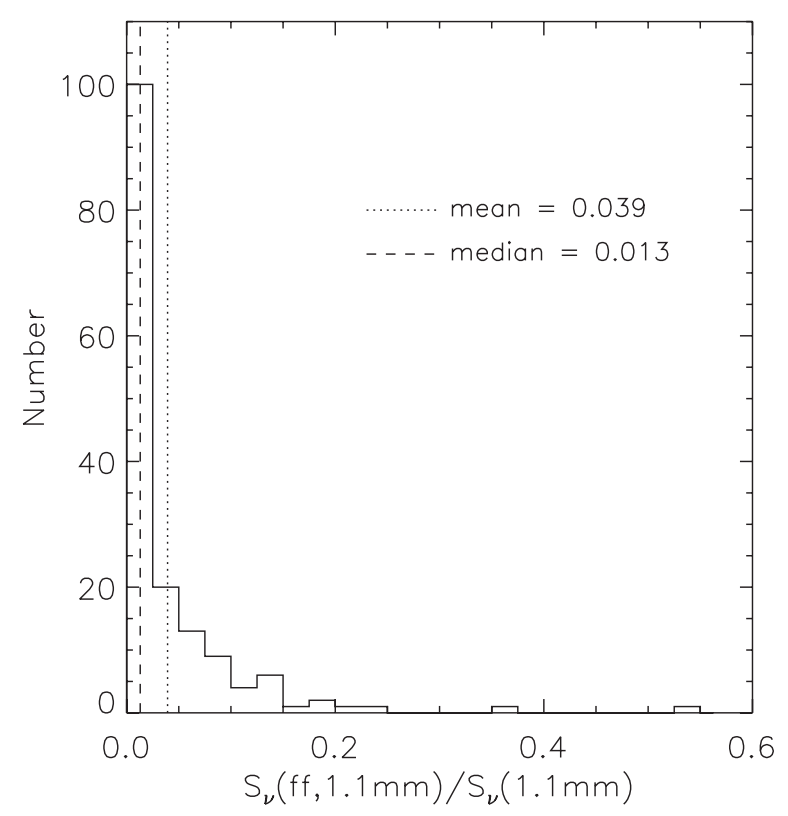

Figure 5. Number of sources as a function of the fraction of $1.1 \mathrm{~mm}$ flux density which is due to free-free emission based on $6 \mathrm{~cm}$ radio continuum observations toward RMS sources (Urquhart et al. 2009a) as discussed in Section 4.1. The mean fraction of flux density attributed to free-free emission is 0.039 and the median is 0.013 . Overall, free-free emission is not the cause of the trend of increasing flux density seen with increasing probability of star formation, although for some BGPS sources the free-free contribution can be significant $(>50 \%)$.

contributions are 0.0002 and 0.54 , respectively, and both values are for BGPS sources in the "starless" group. The RMS $6 \mathrm{~cm}$ observations were targeted toward potential H II regions with the goal of further classifying the RMS sources; some RMS sources were classified as planetary nebulae, which we did not consider as a positive sign of star formation and could therefore fall into our "starless" probability group. The vast majority of the BGPS sources including $6 \mathrm{~cm}$ emission fall in probability group 3, with a mean free-free contribution of $0.033 \pm 0.046$, median of 0.012 , and a maximum of 0.23 . For all but four of the 159 BGPS sources with $6 \mathrm{~cm}$ radio observations, contamination from free-free emission does not exceed $20 \%$, while for the remaining four BGPS sources the free-free emission contributes up to approximately half of the $1.1 \mathrm{~mm}$ flux density.

Without radio observations for all BGPS sources, we are unable to correct for the free-free contamination. To assess whether the mean level of free-free contamination we see toward the 159 BGPS sources could be the cause of the increasing flux density with probability of star formation, we calculate the fraction of the group 1-3 flux that would need to be attributed to free-free emission for the mean flux density to match the mean of the "starless" group. We find 52\%, 64\%, and $91 \%$ for groups $1-3$, respectively. This is an order of magnitude higher than the mean free-free flux density contribution we find with the RMS $6 \mathrm{~cm}$ observations. Therefore, it is highly unlikely that the trend of increasing flux density is due to free-free contamination. However, free-free emission could contribute significantly to the flux densities of individual sources.

The observed increase in flux density with group could also be a reflection of an increasing dust temperature. While an increase in $T_{D}$ does explain some of the observed trends in flux density, it cannot explain the order of magnitude increase in mean flux density between groups 0 and 3 . We discuss the effects of $T_{D}$ in Section 4.2 below.
A SIMBAD search of the 22 "starless" sources in the inner Galaxy with $S_{v}>10$ Jy revealed that only four of the brightest BGPS sources are possibly truly starless, while the remaining 18 brightest BGPS sources in the inner Galaxy had a combination of radio emission and masers as indicators of active star formation. This accentuates that while these BGPS sources are "starless" in the sense that they do not contain any mid-IR sources from GLIMPSE or RMS catalogs, they are not necessarily truly starless sources. Further comparison to other surveys will be needed in order to find a truly starless sample of BGPS sources.

Figure 6 shows the number of BGPS sources as a function of the concentration parameter in each star formation probability group. The concentration parameter is defined as the ratio of the elliptical radius containing $90 \%$ of the total source flux to the elliptical radius containing $50 \%$ of the total source flux, where the elliptical radius is given by $R=\left(R_{\text {maj }} R_{\text {min }}\right)^{1 / 2}$. By this definition, compact sources will have a low concentration parameter while sources with large skirts of emission will have a high concentration parameter. The "starless" sources have a lower mean concentration parameter, and the mean value increases with probability of star formation activity. The majority of the most extended BGPS sources fall within probability group 3 . These statistics are likely affected by chance alignments since more extended sources are more likely to be identified by chance alignments with the mid-IR sources. Since we cannot identify which BGPS-mid-IR source associations are due to chance alignments, we cannot assess the extent of the effect of chance alignments on this trend. However, we do note that the group 3 sources are unlikely to include many chance alignments since we expect a very low fraction of matched EGO and RMS sources to be the result of chance alignments.

Another possible bias which could affect the statistics of each probability group is distance. Due to the limited sensitivity of the mid-IR catalogs, it is possible that more distant BGPS sources harbor low-mass YSOs which are not present in the mid-IR catalogs. The starless group could include a large number of distant BGPS sources which are actually forming stars.

\subsection{Physical Properties}

We create a distance subsample of BGPS sources within the region where all surveys overlap that have distance estimates from a molecular line study of BGPS sources (Schlingman et al. 2011) and very long baseline interferometry (VLBI) parallax measurements (seven BGPS sources; Bartkiewicz et al. 2008; Brunthaler et al. 2009; Xu et al. 2009). Schlingman et al. (2011) used the VLBI parallax based model of Galactic rotation of Reid et al. (2009) and were able to assign the near kinematic distance to sources based on the association with a GLIMPSE IRDC (Peretto \& Fuller 2009). Schlingman et al. (2011) additionally adopted distances from the well-studied sample of massive star-forming regions of Shirley et al. (2003). The "distance subsample" presented in this section consists of 280 BGPS sources which are located in the region where all the catalogs overlap. Since the dominant number of distance determinations are based on the association of a BGPS source with an IRDC, the distance subsample is significantly biased to the near kinematic distance in the inner Galaxy. Full results of the molecular line study are formally presented in Schlingman et al. (2011).

We calculate properties such as mass, volume-averaged density, and column density for the 280 BGPS sources with kinematic distances. The distance subsample samples the different probability groups well with 55, 67, 97, and 61 from groups $0-3$, respectively. Figure 7 plots a histogram of the distances 


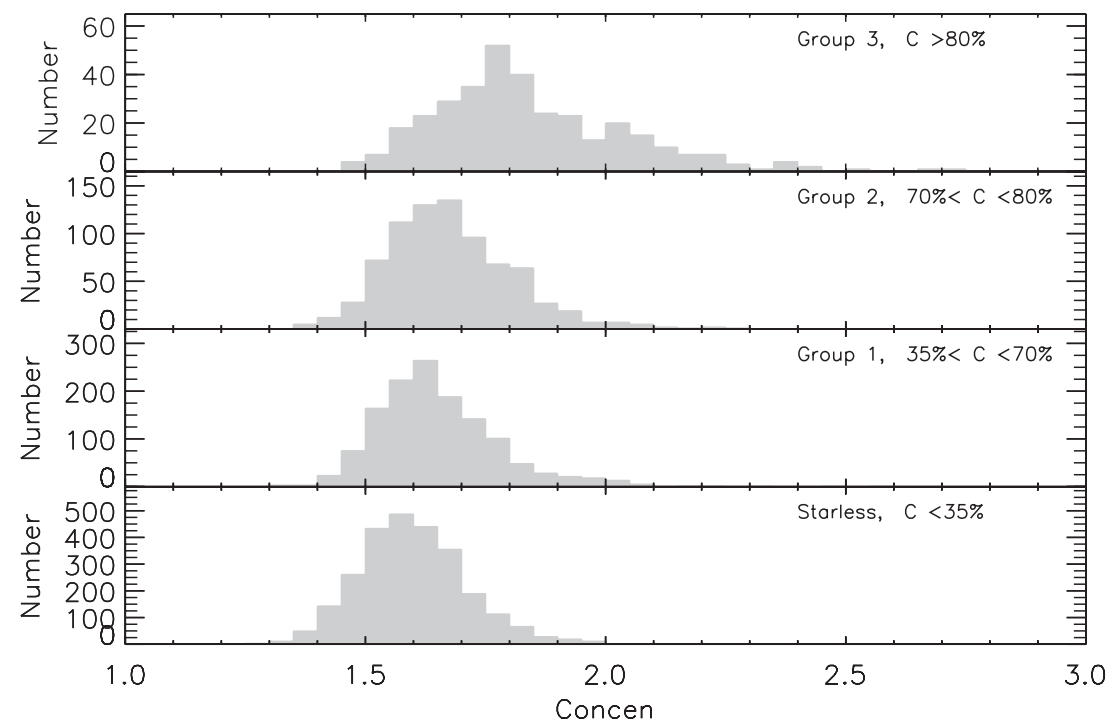

Figure 6. Number of sources as a function of the BGPS concentration parameter for the different probability groups of BGPS sources. Only the 5067 BGPS sources in the region where all catalogs overlap are included. The concentration parameter is given by the ratio of the elliptical radius containing $90 \%$ of the millimeter emission to the elliptical radius including $50 \%$ of the emission. A smaller concentration parameter means that the source is more compact, while sources with large skirts of emission will have a higher concentration parameter. Note that the vertical scale is different for each probability group. The range of confidence values for each probability group is given in the upper right corner of each panel.

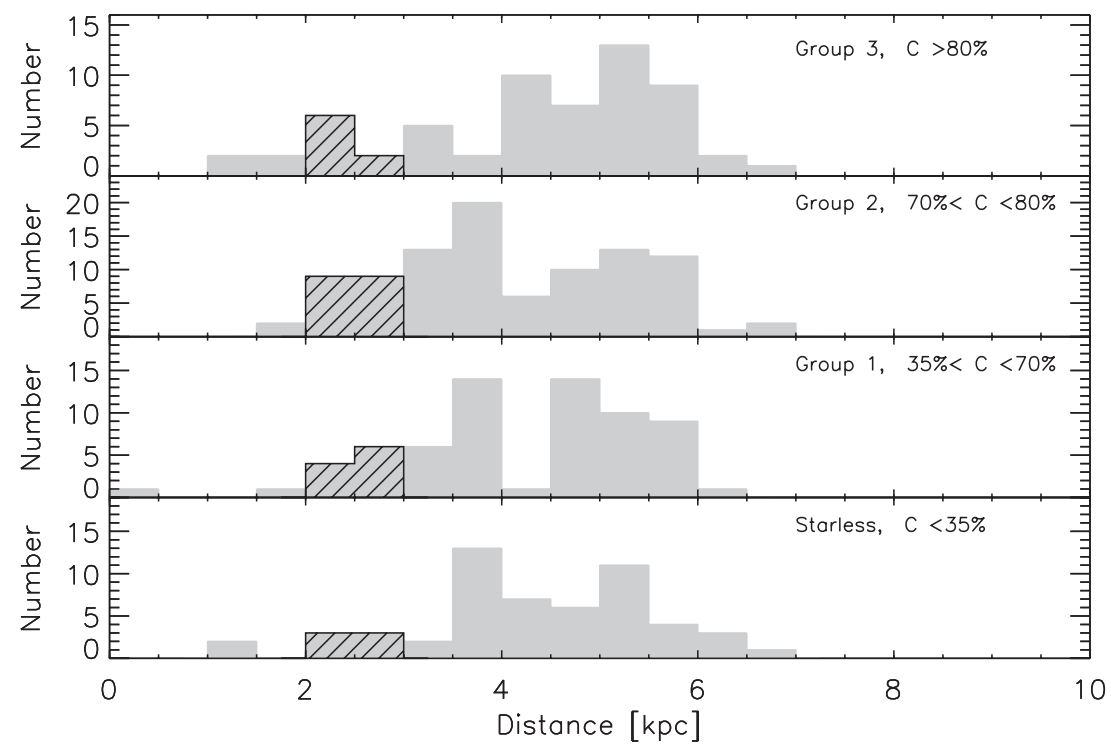

Figure 7. Number of sources as a function of distance for each of the different probability groups of BGPS sources which have distance measurements and are located in the region where all catalogs overlap. The striped histogram represents the $2 \mathrm{kpc}$ subsample. Note that the vertical scale is different for each probability group. The range of confidence values for each probability group is given in the upper right corner of each panel.

as a function of probability of star formation activity. The distances range from 0.21 to $7.0 \mathrm{kpc}$ with no obvious trends with star formation probability (see Table 3 ). Since the BGPS will be sensitive to different structures over such a large range in distances, we define a subsample of sources with $2 \mathrm{kpc}<d<3 \mathrm{kpc}$ (hereafter referred to as the $2 \mathrm{kpc}$ subsample) in order to separate trends from the ambiguity introduced by a large range of distances. We have chosen this range of distances because Dunham et al. (2010) find that at distances greater than approximately $2 \mathrm{kpc}$ the BGPS is sensitive to structures most closely related to clumps. Thus, we expect the $2 \mathrm{kpc}$ subsample to have properties in the typical range of clumps (e.g., Table 1 of Bergin $\&$ Tafalla 2007). We find $6,10,18$, and 8 sources with distances of $2-3 \mathrm{kpc}$ for probability groups $0-3$, respectively.
Figure 8 plots the number of objects versus physical radius for each probability group. The gray histograms show the distance subsample, while the striped histograms mark the $2 \mathrm{kpc}$ subsample. The elliptical radius was determined by the BGPS source extraction software based on the emission-weighted moments of the BGPS maps (Rosolowsky et al. 2010). Radii of unresolved sources have been set to an upper limit of half the beam size, 16.5. The physical radii of sources in the distance subsample range from 0.07 to $3.8 \mathrm{pc}$ spanning the range of sizes expected for the entire hierarchical structure in giant molecular clouds (GMCs) including clouds, clumps, and cores (radius $=1-7.5 \mathrm{pc}, 0.15-1.5$, pc and $0.01-0.1 \mathrm{pc}$, respectively; Bergin \& Tafalla 2007). The radii of the $2 \mathrm{kpc}$ subsample range from 0.18 to $1.4 \mathrm{pc}$, and span the range of typical clump radii 


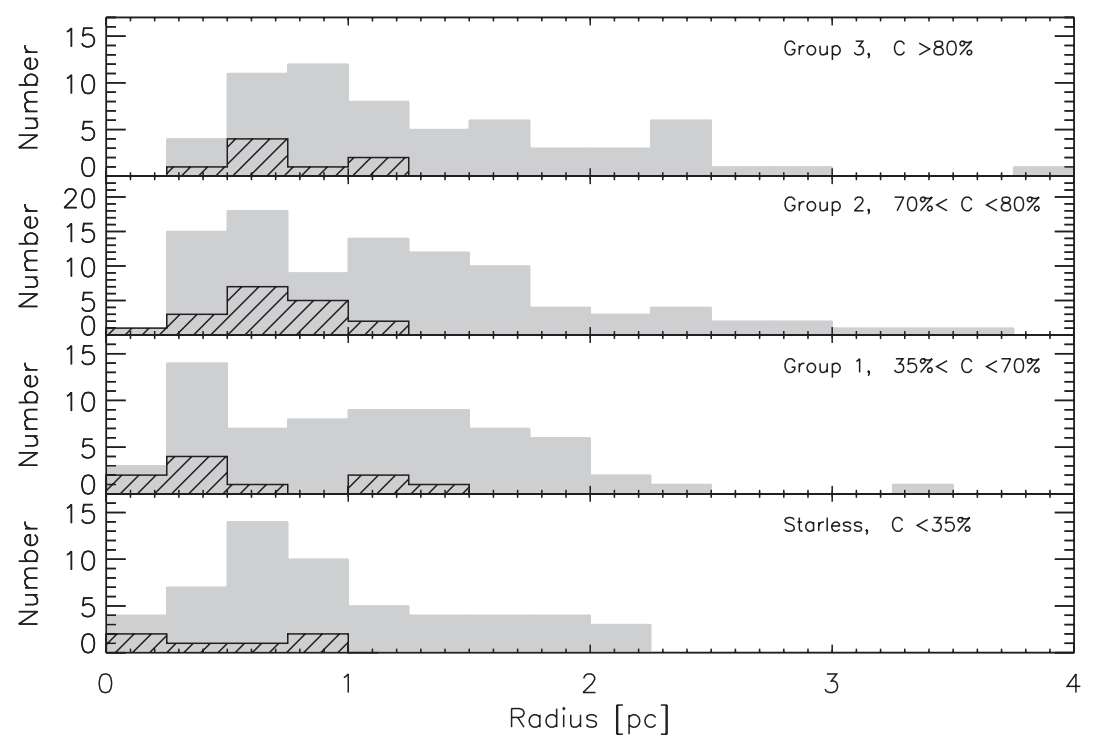

Figure 8. Number of sources as a function of physical radius for each of the probability groups of BGPS sources which have distance measurements and are located in the region where all catalogs overlap. The striped histogram represents the $2 \mathrm{kpc}$ subsample. The range of confidence values for each probability group is given in the upper-right corner of each panel.

(see Table 3). The physical radius increases with star formation probability group from $0.95 \mathrm{pc}$ for the "starless" sources to 1.3 pc for group 3 (see Table 3 ).

We calculate the isothermal dust mass, $M_{\text {iso }}$, assuming a single dust temperature, $T_{D}$, as

$$
\begin{aligned}
M_{\mathrm{iso}}= & \frac{S_{v} D^{2}}{\kappa_{\nu} B_{v}\left(T_{D}\right)}=13.1 M_{\odot}\left(\frac{S_{v}}{1 \mathrm{Jy}}\right)\left(\frac{D}{1 \mathrm{kpc}}\right)^{2} \\
& \times\left(\frac{e^{13.0 \mathrm{~K} / T_{D}}-1}{e^{13.0 \mathrm{~K} / 20.0 \mathrm{~K}}-1}\right)
\end{aligned}
$$

where $S_{v}$ is the flux density, $D$ is the distance, $\kappa_{v}$ is the dust opacity per gram of gas and dust including a gas-to-dust ratio of $100\left(\kappa_{v}=0.0114 \mathrm{~cm}^{2} \mathrm{~g}^{-1}\right.$ at $271.1 \mathrm{GHz}$; logarithmically interpolated from $\mathrm{OH} 5$ dust from Column 5 of Table 1 in Ossenkopf \& Henning 1994), and $B_{v}$ is the Planck function evaluated at $T_{D}$. The right-hand side of the equation is evaluated for $T_{D}=20 \mathrm{~K}$ based on $\mathrm{NH}_{3}$ observations of BGPS sources in the Gemini OB1 molecular cloud where a mean gas kinetic temperature of $20 \mathrm{~K}$ was found (Dunham et al. 2010).

Although $20 \mathrm{~K}$ is a reasonable value of $T_{D}$ to assume if no other information is available, we have both gas kinetic temperature and dust temperature measurements from other observations. M. K. Dunham et al. (2011, in preparation) have surveyed 716 BGPS sources in the inner Galaxy in $\mathrm{NH}_{3}(1,1)$, $(2,2)$, and $(3,3)$ and have provided a measurement of $T_{K}$ toward the 408 BGPS sources detected in $\mathrm{NH}_{3}$. They find $\left\langle T_{K}\right\rangle$ of $14.0 \pm 3.2 \mathrm{~K}, 14.8 \pm 3.9 \mathrm{~K}, 16.1 \pm 4.3 \mathrm{~K}$, and $22.0 \pm 5.9 \mathrm{~K}$ based on $130,143,102$, and 33 BGPS sources from groups $0-3$, respectively. For this work, we assume the mean gas kinetic temperatures from the $\mathrm{NH}_{3}$ study for each group. By assuming that the gas kinetic temperature is equivalent to the dust temperature, we are assuming that the density is high enough to bring the temperatures into equilibrium via collisions. This assumption holds in the dense regions where the dust effectively shields the gas from the interstellar radiation field (ISRF) and the gas is heated via collisions with the dust (e.g., Goldreich \& Kwan 1974; Evans et al. 2001).
The number of BGPS sources of each $M_{\text {iso }}$ are plotted for each probability group in Figure 9, where the gray histogram represents the entire distance subsample and the striped histogram denotes the $2 \mathrm{kpc}$ subsample. There is a clear trend of increasing mass with increasing probability of star formation activity; the median masses in groups 0-3 are 340, 690, 680, and $1160 M_{\odot}$, respectively. Similarly, we find a median mass of $52,94,170,710 M_{\odot}$ for groups $0-3$ of the $2 \mathrm{kpc}$ subsample, respectively. The median mass is a more accurate representation of each group than the mean mass due to the few extremely high mass $\left(10^{4} M_{\odot}\right)$ BGPS sources in groups 0,2 , and 3 (see Table 3).

There are two effects that could artificially produce the observed trend in mass: contamination by free-free emission and the assumed $T_{D}$. As discussed in Section 4.1, the free-free emission is not a significant contribution to the flux densities of most BGPS sources and is unlikely to cause the trend of increasing mass.

Here we explore what effects different dust temperatures would have on the observed trend in mass. We calculate the temperature required for the median mass of each group to equal the median mass of group 1 (where we assume $T_{D}=14.8 \mathrm{~K}$ ). We have chosen to use the median mass of group 1 as the standard since it is very similar to the median for group 2 (690 and $680 M_{\odot}$, respectively). Dust temperatures of 9.2, 15.9, and $33.2 \mathrm{~K}$ would bring the median of groups 0,2 , and 3 into alignment with group 1 , respectively. While these temperatures are reasonable, the values for groups 0 and 3 lie outside of the standard deviation of the mean gas kinetic temperatures derived from the $\mathrm{NH}_{3}$ observations discussed above. The $\mathrm{NH}_{3}$ observations are sensitive to gas kinetic temperatures well above $33 \mathrm{~K}$, so the lower mean temperature observed toward group 3 sources is not the result of an observational bias.

Thus, the trend to higher masses with probability of star formation activity is likely real. Here $M_{\text {iso }}$ ranges from $2.4 M_{\odot}$ to $3.6 \times 10^{4} M_{\odot}$ for the distance subsample, and $19 M_{\odot}$ to $2.5 \times 10^{3} M_{\odot}$ for the $2 \mathrm{kpc}$ subsample. Again, the distance subsample spans the range of masses for cores, clumps, and clouds $\left(0.5-5 M_{\odot}, 50-500 M_{\odot}\right.$, and $10^{3}-10^{4} M_{\odot}$, respectively; 
Table 3

Physical Property Statistics of BGPS Sources in the Region Where All Catalogs Overlap

\begin{tabular}{|c|c|c|c|c|c|c|}
\hline $\begin{array}{l}\text { Property } \\
\text { (units) }\end{array}$ & $\begin{array}{l}\text { Probability } \\
\text { Group }\end{array}$ & Minimum & Mean & $\begin{array}{l}\text { Standard } \\
\text { Deviation }\end{array}$ & Median & Maximum \\
\hline$S_{v}$ & 0 & 0.06 & 0.71 & 1.4 & 0.41 & 33.1 \\
\hline \multirow[t]{3}{*}{ (Jy) } & 1 & 0.06 & 1.5 & 1.9 & 0.89 & 27.7 \\
\hline & 2 & 0.08 & 2.0 & 2.5 & 1.2 & 25.3 \\
\hline & 3 & 0.13 & 8.0 & 18.0 & 3.4 & 146.8 \\
\hline \multirow{4}{*}{$\begin{array}{l}\text { Concentration } \\
\text { parameter }\end{array}$} & 0 & 1.3 & 1.6 & 0.11 & 1.6 & 2.1 \\
\hline & 1 & 1.3 & 1.7 & 0.13 & 1.6 & 2.6 \\
\hline & 2 & 1.4 & 1.7 & 0.13 & 1.7 & 2.3 \\
\hline & 3 & 1.5 & 1.8 & 0.20 & 1.8 & 2.7 \\
\hline \multirow{4}{*}{$\begin{array}{l}N_{\mathrm{H}_{2}}^{\text {beam }} \\
\left(10^{22} \mathrm{~cm}^{-2}\right)\end{array}$} & 0 & 0.21 & 0.95 & 1.2 & 0.71 & 23.3 \\
\hline & 1 & 0.20 & 1.4 & 1.7 & 0.95 & 27.0 \\
\hline & 2 & 0.22 & 1.6 & 1.6 & 1.1 & 19.3 \\
\hline & 3 & 0.20 & 4.7 & 10.4 & 2.1 & 99.3 \\
\hline \multicolumn{7}{|c|}{ Distance Subsample } \\
\hline \multirow{4}{*}{$\begin{array}{l}\text { Distance } \\
(\mathrm{kpc})\end{array}$} & 0 & 1.04 & 4.3 & 1.2 & 4.4 & 6.7 \\
\hline & 1 & 0.21 & 4.2 & 1.2 & 4.5 & 6.4 \\
\hline & 2 & 1.9 & 4.1 & 1.2 & 3.8 & 7.0 \\
\hline & 3 & 1.3 & 4.4 & 1.4 & 4.6 & 7.0 \\
\hline \multirow{4}{*}{$\begin{array}{l}\text { Radius } \\
\text { (pc) }\end{array}$} & 0 & 0.12 & 0.95 & 0.55 & 0.86 & 2.0 \\
\hline & 1 & 0.07 & 1.1 & 0.63 & 1.0 & 3.4 \\
\hline & 2 & 0.18 & 1.2 & 0.75 & 1.1 & 3.5 \\
\hline & 3 & 0.35 & 1.3 & 0.72 & 1.1 & 3.8 \\
\hline \multirow{4}{*}{$\begin{array}{l}M_{\text {iso }}\left(T_{D}\right) \\
\left(M_{\odot}\right)\end{array}$} & 0 & 19 & 1050 & 1720 & 340 & $1.0 \times 10^{4}$ \\
\hline & 1 & 2.4 & 1040 & 1130 & 690 & 5380 \\
\hline & 2 & 30 & 1450 & 2110 & 680 & $1.2 \times 10^{4}$ \\
\hline & 3 & 90 & 3230 & 5450 & 1160 & $3.6 \times 10^{4}$ \\
\hline \multirow{4}{*}{$\begin{array}{l}n \\
\left(10^{3} \mathrm{~cm}^{-3}\right)\end{array}$} & 0 & 0.73 & 7.1 & 11.0 & 3.3 & 56.1 \\
\hline & 1 & 0.32 & 6.7 & 9.4 & 2.5 & 50.2 \\
\hline & 2 & 0.41 & 5.8 & 10.7 & 2.5 & 85.7 \\
\hline & 3 & 0.60 & 7.2 & 9.7 & 3.2 & 45.8 \\
\hline \multirow{4}{*}{$\begin{array}{l}N_{\mathrm{H}_{2}}^{\text {avg }} \\
\left(10^{21} \mathrm{~cm}^{-2}\right)\end{array}$} & 0 & 3.1 & 12.8 & 9.7 & 9.6 & 56.3 \\
\hline & 1 & 3.7 & 12.8 & 9.9 & 9.7 & 47.8 \\
\hline & 2 & 3.2 & 12.4 & 10.8 & 9.2 & 82.2 \\
\hline & 3 & 3.4 & 24.2 & 31.3 & 12.6 & 154.0 \\
\hline \multicolumn{7}{|c|}{2 kpc Subsample } \\
\hline \multirow{4}{*}{$\begin{array}{l}\text { Radius } \\
\text { (pc) }\end{array}$} & 0 & 0.19 & 0.49 & 0.29 & 0.51 & 0.90 \\
\hline & 1 & 0.19 & 0.65 & 0.41 & 0.49 & 1.4 \\
\hline & 2 & 0.18 & 0.67 & 0.27 & 0.65 & 1.1 \\
\hline & 3 & 0.41 & 0.74 & 0.23 & 0.73 & 1.1 \\
\hline \multirow{4}{*}{$\begin{array}{l}M_{\text {iso }}\left(T_{D}\right) \\
\left(M_{\odot}\right)\end{array}$} & 0 & 37 & 79 & 56 & 52 & 179 \\
\hline & 1 & 26 & 390 & 767 & 94 & 2520 \\
\hline & 2 & 19 & 192 & 130 & 170 & 546 \\
\hline & 3 & 90 & 792 & 609 & 710 & 2120 \\
\hline \multirow{4}{*}{$\begin{array}{l}n \\
\left(10^{3} \mathrm{~cm}^{-3}\right)\end{array}$} & 0 & 1.0 & 8.2 & 11.3 & 3.6 & 29.5 \\
\hline & 1 & 0.85 & 5.3 & 5.8 & 3.7 & 16.3 \\
\hline & 2 & 0.91 & 4.0 & 4.2 & 2.3 & 14.2 \\
\hline & 3 & 2.0 & 9.1 & 6.9 & 8.1 & 22.3 \\
\hline \multirow{4}{*}{$\begin{array}{l}N_{\mathrm{H}_{21}}^{\mathrm{avg}} \\
\left(10^{21} \mathrm{~cm}^{-2}\right)\end{array}$} & 0 & 2.7 & 7.2 & 6.8 & 4.7 & 19.9 \\
\hline & 1 & 2.4 & 7.9 & 6.9 & 6.1 & 25.4 \\
\hline & 2 & 3.1 & 6.4 & 3.5 & 5.0 & 14.5 \\
\hline & 3 & 6.3 & 21.0 & 13.7 & 20.4 & 44.6 \\
\hline
\end{tabular}

Bergin \& Tafalla 2007), while the $2 \mathrm{kpc}$ subsample encompasses the mass range for clumps without extending into the range for clouds or cores.

From $M_{\text {iso }}$ we calculate the mean particle density, $n$, for each source in the distance subsample via $n=3 M_{\text {iso }} / 4 \pi R^{3}$, where $R$ is the source radius determined by the BGPS source extraction algorithm. This density calculation assumes a constant density and the effect is a volume-averaged density that is more reflective of the lower density outer regions of the BGPS source than the higher density regions. Figure 10 shows histograms of $n$ for each probability group. All groups span almost the same range from 320 to $5.6 \times 10^{4} \mathrm{~cm}^{-3}$. However, the BGPS 


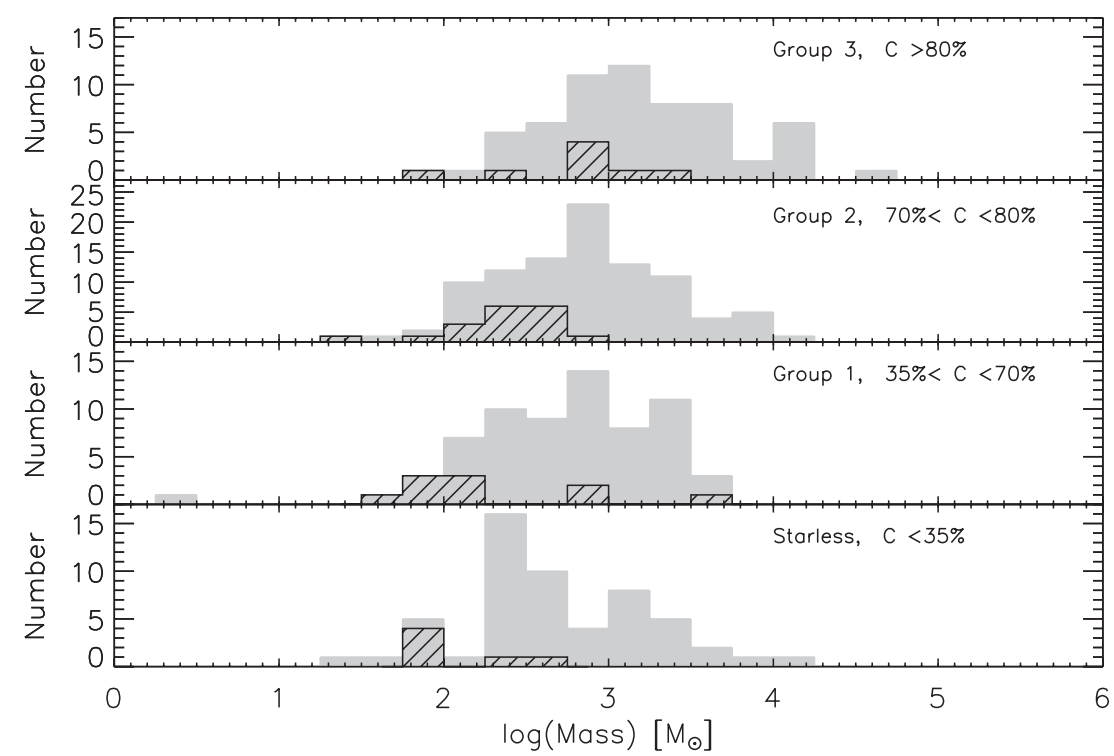

Figure 9. Number of sources as a function of isothermal mass for each of the probability groups of BGPS sources which have distance measurements and are located in the region where all catalogs overlap. The striped histogram represents the $2 \mathrm{kpc}$ subsample. Note that the vertical scale is different for each probability group. The range of confidence values for each probability group is given in the upper right corner of each panel.

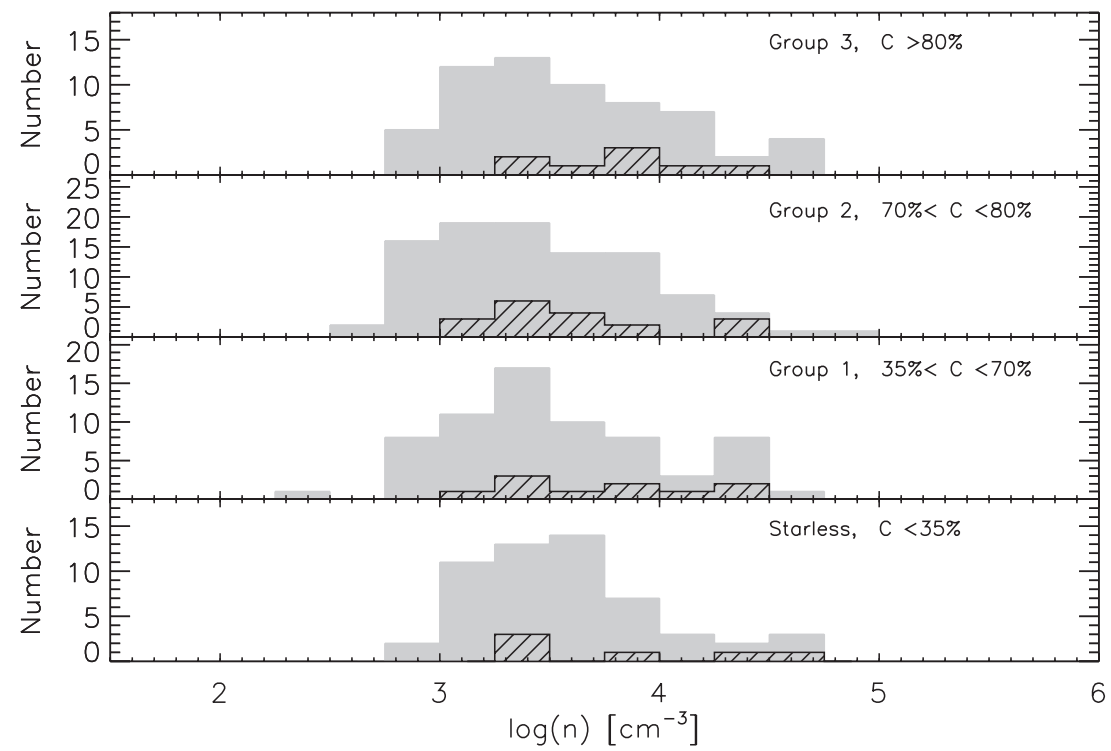

Figure 10. Number of sources as a function of mean volume-averaged density for the subset of each BGPS source probability group which have distance measurements and are located in the region where all catalogs overlap. The striped histogram represents the $2 \mathrm{kpc}$ subsample. Note that the vertical scale is different for each probability group. The range of confidence values for each probability group is given in the upper right corner of each panel.

sources in groups 0 and 3 have, on average, a higher density than those in groups 1 and 2 (see Table 3). The "starless" sources are not only less massive but also smaller than BGPS sources in groups 1-3 and therefore have densities similar to those of group 3 . The $2 \mathrm{kpc}$ subsample only extends to about $10^{3} \mathrm{~cm}^{-3}$ on the low density end of each distribution, but does extend to $10^{4} \mathrm{~cm}^{-3}$ on the high density end, even in the "starless" category. Clouds typically range from 50 to $500 \mathrm{~cm}^{-3}$, clumps from $10^{3}$ to $10^{4} \mathrm{~cm}^{-3}$, and cores from $10^{4}$ to $10^{5} \mathrm{~cm}^{-3}$ (Bergin \& Tafalla 2007). Overall, the distance subsample spans the entire range of densities, but the $2 \mathrm{kpc}$ subsample only spans the density ranges for clumps and cores.

The column density of $\mathrm{H}_{2}$ is given by $N_{\mathrm{H}_{2}}^{\text {avg }}=$ $M_{\text {iso }} / 4 \pi \mu m_{\mathrm{H}} R_{\mathrm{obj}}^{2}$, where $\mu=2.37$ and $m_{\mathrm{H}}$ is the mass of hydrogen. Figure 11 shows the number of sources as a function of $N_{\mathrm{H}_{2}}^{\text {avg }}$ for each probability group in the distance subsample (gray histogram) as well as for the $2 \mathrm{kpc}$ subsample (striped histogram). There is an increase in mean $N_{\mathrm{H}_{2}}^{\text {avg }}$ between groups $0-2$ and group 3. Groups 0-2 have mean $N_{\mathrm{H}_{2}}^{\text {avg }}$ values near $1.2 \times 10^{22} \mathrm{~cm}^{-2}$ while group 3 has a mean $\mathrm{H}_{2}$ column density of $2.4 \times 10^{22} \mathrm{~cm}^{-2}$ (see Table 3). The dashed line denotes the threshold column density of about $123 M_{\odot} \mathrm{pc}^{-2}$ which has been observed toward nearby, low-mass star-forming regions (Lada et al. 2010; Heiderman et al. 2010). The dotted line denotes the theoretical minimum surface density, $1 \mathrm{~g} \mathrm{~cm}^{-2}$, required to prevent fragmentation and allow a massive star to form (Krumholz \& McKee 2008). Similar to the volume density, $N_{\mathrm{H}_{2}}^{\text {avg }}$ is also an average quantity. There are likely higher surface density regions within the BGPS source where the YSOs are forming, but the BGPS is sensitive to the lower density outer regions of the clump (Dunham et al. 2010). 


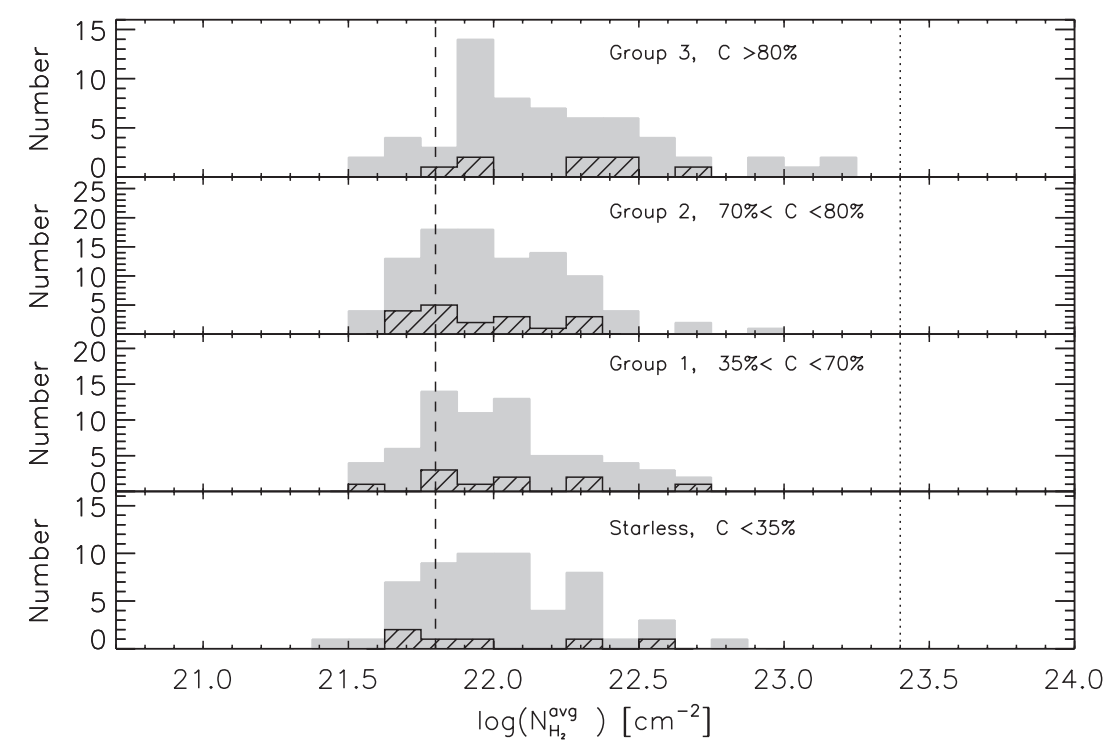

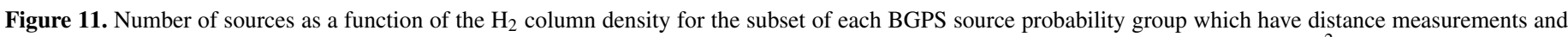

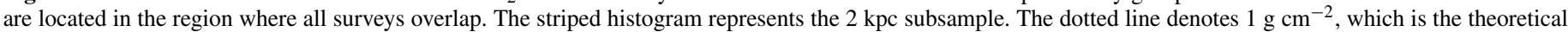

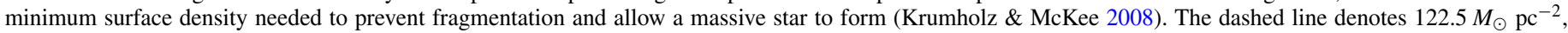

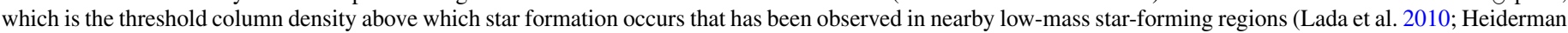

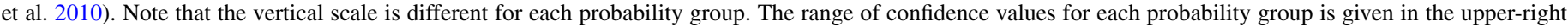
corner of each panel.

This is consistent with the picture that RMS and EGO sources are high-mass YSOs even though the average surface density is not above the $1 \mathrm{~g} \mathrm{~cm}^{-2}$ requirement to prevent fragmentation.

Alternatively, we can calculate the $\mathrm{H}_{2}$ column density per beam for all BGPS sources within the area where all catalogs overlap since this quantity is independent of distance. The $\mathrm{H}_{2}$ column density per beam is given by

$$
N_{\mathrm{H}_{2}}^{\text {beam }}=\frac{S_{v}\left(40^{\prime \prime}\right)}{\Omega_{\text {beam }} \mu_{\mathrm{H}_{2}} m_{\mathrm{H}} \kappa_{v} B_{v}\left(T_{D}\right)},
$$

where $S_{v}\left(40^{\prime \prime}\right)$ is the $1.1 \mathrm{~mm}$ flux density within an aperture with a diameter of $40^{\prime \prime}, \Omega_{\text {beam }}$ is the solid angle of the beam, $\mu_{\mathrm{H}_{2}}=2.8, \kappa_{v}$ is the dust opacity and $B_{v}\left(T_{D}\right)$ is the Planck function evaluated at the dust temperature, $T_{D}$, as described above. We use $S_{v}\left(40^{\prime \prime}\right)$ as a measure of the flux within a beam since a top-hat function with a $20^{\prime \prime}$ radius has the same solid angle as a Gaussian beam with an FWHM of $33^{\prime \prime}$. We have applied an aperture correction of $1.46 \pm 0.05$ to $S_{v}\left(40^{\prime \prime}\right)$ to account for power that falls outside of the $40^{\prime \prime}$ aperture due to the sidelobes of the CSO beam (Aguirre et al. 2011). We calculate this property for all BGPS sources within the region where all surveys overlap, since $N_{\mathrm{H}_{2}}^{\text {beam }}$ is independent of distance. Figure 12 shows the distribution of $\mathrm{H}_{2}$ column density per beam for the BGPS sources in the overlap region for each probability group. As above, the dotted line denotes the column density required to prevent fragmentation (Krumholz \& McKee 2008) and the dashed line denotes the threshold for efficient star formation (Lada et al. 2010; Heiderman et al. 2010). The mean column density per beam increases with probability group with $\left\langle N_{\mathrm{H}_{2}}^{\text {beam }}\right\rangle=0.95 \times 10^{22}, 1.4 \times 10^{22}, 1.6 \times 10^{22}$, and $4.7 \times$ $10^{22} \mathrm{~cm}^{-2}$ for groups $0-3$, respectively. The median $N_{\mathrm{H}_{2}}^{\text {beam }}$ of group $0\left(7.1 \times 10^{21} \mathrm{~cm}^{-2}\right)$ is similar to the threshold for efficient star formation $\left(6.5 \times 10^{21} \mathrm{~cm}^{-2}\right.$; dashed line in Figure 12; Lada et al. 2010; Heiderman et al. 2010), such that roughly half of the "starless" BGPS sources have a peak column density high enough to efficiently form stars. It may be that these sources appear "starless" in this study because of the sensitivity limits of the mid-IR catalogs. For example, low-mass YSOs within a BGPS source at $5 \mathrm{kpc}$ would not be detected by the GLIMPSE surveys. Further comparisons with other tracers of star formation are necessary to determine which sources are truly starless.

Overall, we find that $S_{v}$ (int), $R, M_{\text {iso }}, N_{\mathrm{H}_{2}}^{\text {beam }}$, and $N_{\mathrm{H}_{2}}^{\text {avg }}$ are all higher in BGPS sources actively forming stars. Sources identified as actively forming stars are typically extended with large skirts of emission, while the "starless" sources are more compact, although this trend is likely affected by chance alignments since more extended BGPS sources are more likely to be matched with a mid-IR source by chance. BGPS sources with EGO or RMS sources (group 3) are more massive and have higher volume-averaged densities than BGPS sources with GLIMPSE sources. As expected, due to the large range of distances, these physical properties show that the BGPS sources span the entire hierarchical structure in GMCs (clouds, clumps, and cores). Based on the physical properties presented, we confirm that the $2 \mathrm{kpc}$ subsample is mostly composed of clumps.

\section{SUMMARY}

We have cross-matched the BGPS catalog with available mid-infrared Galactic plane catalogs to determine which BGPS sources show signs of active star formation. The mid-infrared catalogs include the GLIMPSE Red Source catalog (R08), the EGO catalog (Cyganowski et al. 2008), and the RMS catalog (Hoare et al. 2004). We have also created an additional GLIMPSE Red Source catalog with a less stringent color selection than that used by R08, namely [4.5] - [8.0] $>0.75$.

Overall, 3712 BGPS sources (44\%) include at least one mid-IR source. Within the area where all surveys overlap $\left(10^{\circ}<\ell<65^{\circ}\right), 2457$ of 5067 (48\%) BPGS sources include at least one mid-IR source. If we account for the number of expected chance alignments between the mid-IR and BGPS catalogs, we estimate the total number of BGPS sources showing mid-IR signs of active star formation to be 1472 over the whole 


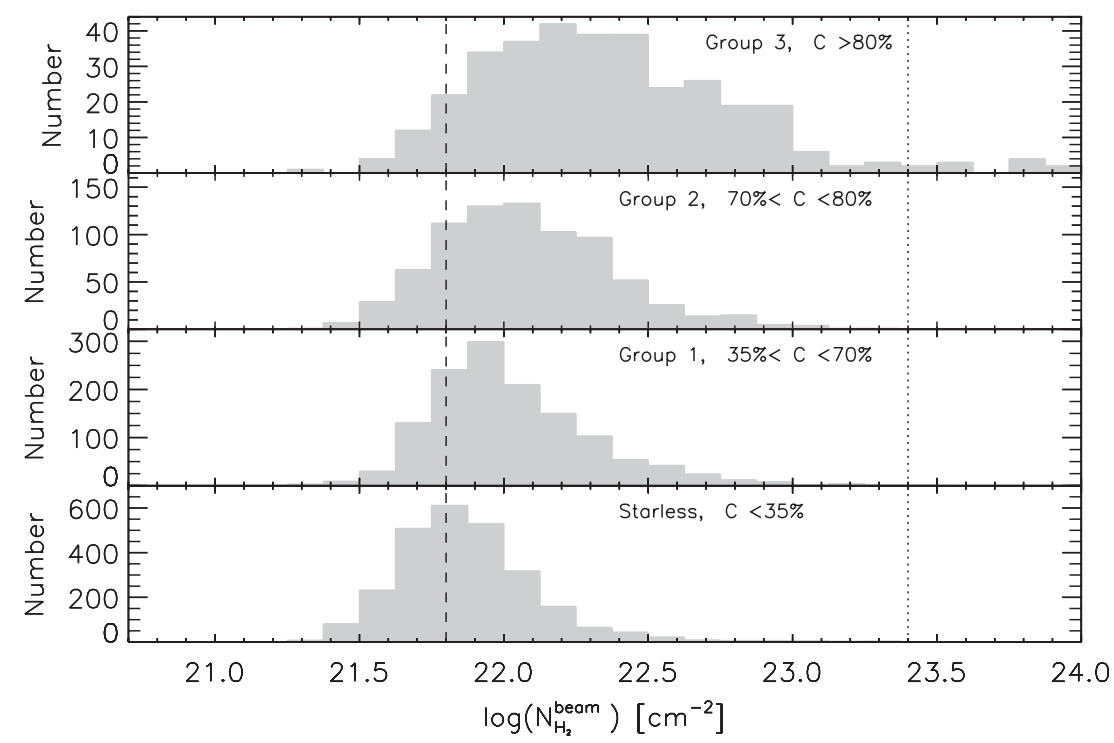

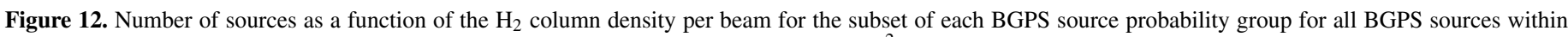

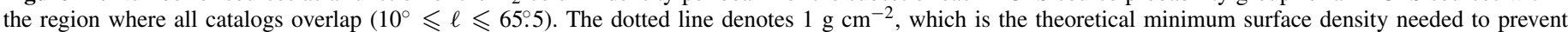

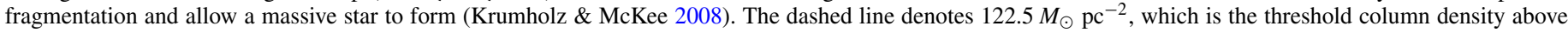

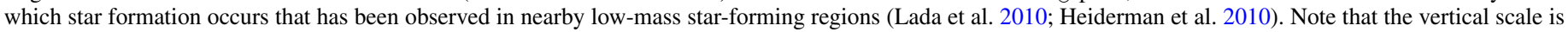
different for each probability group. The range of confidence values for each probability group is given in the upper right corner of each panel.

survey $(17.6 \%)$ and 1035 within the area where all surveys overlap (20.4\%). These low percentages are conservative lower limits, while the true fraction of BGPS sources forming stars is likely higher.

We additionally divide the BGPS sources into four groups representing the probability of star formation activity based on the highest confidence parameter mid-IR source matched to each BGPS source in order to study physical properties as a function of star formation probability. The sources with the highest probability of star formation activity are BGPS sources with an EGO or young RMS source ( $90 \%$ confidence). The lowest probability group corresponds to sources which were not matched with any mid-IR sources and are considered to be "starless" in this picture.

The mean BGPS flux increases with probability of star formation activity, with the BGPS sources matched to EGOs and young RMS sources statistically having the highest fluxes. The distribution of "starless" sources has the lowest mean flux but does include a tail up to $\sim 30 \mathrm{Jy}$. While BGPS sources without mid-IR associations are designated "starless" here, a literature search shows that these bright sources do actually show signs of active star formation in other tracers, such as masers, $\mathrm{X}$-ray emission, and radio emission, which demonstrates the importance of including other star formation tracers when defining a truly starless sample. For example, these "starless" sources may harbor young, highly embedded YSOs which only exhibit emission at wavelengths longward of $24 \mu \mathrm{m}$. A future paper will compare the BGPS catalog with the Spitzer MIPSGAL catalog of 24, 70, and $160 \mu \mathrm{m}$ sources. Additionally, this literature search suggests that the fraction of BGPS sources with active star formation is likely higher than our conservative lower limit of $18 \%$.

The most compact BGPS sources are typically found to be "starless," while BGPS sources with the highest probability of active star formation are typically more extended with large skirts of emission.

We define a sample of 280 sources with kinematic distance determinations ranging from 0.21 to $7.0 \mathrm{kpc}$, which are strongly biased to the near distance in the inner Galaxy. Since BGPS emission arising from $7 \mathrm{kpc}$ will trace a different average density of gas than that arising from $1 \mathrm{kpc}$, we define a subsample of sources with distances between 2 and $3 \mathrm{kpc}$. We see a trend of increasing mass with increasing probability of star formation activity, both in the distance sample and the $2 \mathrm{kpc}$ subsample. Similarly, there is a trend in $\mathrm{H}_{2}$ column density, with the highest mean column density found in the group with the highest probability of star formation.

The physical properties presented show that the BGPS sources span the entire hierarchical structure in GMCs (clouds, clumps, and cores), as expected due to the large range of distances. We also confirm that the $2 \mathrm{kpc}$ subsample is mostly composed of clumps. On average, BGPS sources matched with EGO or young RMS sources are more massive and denser than BGPS sources matched with GLIMPSE red sources or not matched with any mid-IR sources.

The authors thank the anonymous referee for insightful comments which have improved this work. We also thank C. Battersby for providing detailed comments. T.P.R. was supported through the University of Texas at Austin Tinsley Visiting Scholar Program and also by NASA through the Spitzer Space Telescope Fellowship Program, through a contract issued by the Jet Propulsion Laboratory, California Institute of Technology, under a contract with NASA. The BGPS is supported by the National Science Foundation through NSF grant AST-0708403. M.K.D. and N.J.E. were supported by NSF grant AST-0607793 to the University of Texas at Austin. M.K.D. was additionally supported by a grant from the National Radio Astronomy Observatory (NRAO) Student Observing Support Program, award number GSSP09-0004. The NRAO is a facility of the National Science Foundation, operated under cooperative agreement by Associated Universities, Inc. C.J.C. acknowledges support from NSF grant AST-0808119. C.J.C. was partially supported during this work by a NSF Graduate Research Fellowship, and is currently supported by an NSF Astronomy and Astrophysics Postdoctoral Fellowship under award AST-1003134. This paper 
made use of information from the Red MSX Source (RMS) survey database at http://www.ast.leeds.ac.uk/RMS which was constructed with support from the Science and Technology Facilities Council of the UK. This research has made use of NASA Astrophysics Data System (ADS) Abstract Service and of the SIMBAD database, operated at CDS, Strasbourg, France.

\section{REFERENCES}

Aguirre, J. E., et al. 2011, ApJS, 192, 4

Bartkiewicz, A., Brunthaler, A., Szymczak, M., van Langevelde, H. J., \& Reid, M. J. 2008, A\&A, 490, 787

Benjamin, R. A., et al. 2003, PASP, 115, 953

Bergin, E. A., \& Tafalla, M. 2007, ARA\&A, 45, 339

Buemi, C. S., Umana, G., Trigilio, C., \& Leto, P. 2007, A\&A, 462, 637

Breen, S. L., Ellingsen, S. P., Caswell, J. L., \& Lewis, B. E. 2010, MNRAS, 401, 2219

Brunthaler, A., Reid, M. J., Menten, K. M., Zheng, X. W., Moscadelli, L., \& Xu, Y. 2009, ApJ, 693, 424

Carey, S. J., Clark, F. O., Egan, M. P., Price, S. D., Shipman, R. F., \& Kuchar, T. A. 1998, ApJ, 508, 721

Carey, S. J., et al. 2009, PASP, 121, 76

Chambers, E. T., Jackson, J. M., Rathborne, J. M., \& Simon, R. 2009, ApJS, 181,360

Churchwell, E. 2002, ARA\&A, 40, 27

Churchwell, E., et al. 2009, PASP, 121, 213

Cyganowski, C. J., Brogan, C. L., Hunter, T. R., \& Churchwell, E. 2009, ApJ, 702,1615

Cyganowski, C. J., et al. 2008, AJ, 136, 2391

De Buizer, J. M., Radomski, J. T., Telesco, C. M., \& Piña, R. K. 2005, ApJS, 156,179

Di Francesco, J. 2008, BAAS, 40, 271

Dehaes, S., Groenewegen, M. A. T., Decin, L., Hony, S., Raskin, G., \& Blommaert, J. A. D. L. 2007, MNRAS, 377, 931

Dunham, M. K., et al. 2010, ApJ, 717, 1157

Egan, M. P., Shipman, R. F., Price, S. D., Carey, S. J., Clark, F. O., \& Cohen, M. 1998, ApJ, 494, L199

Elia, D., et al. 2010, A\&A, 518, L97

Ellingsen, S. P., Voronkov, M. A., Cragg, D. M., Sobolev, A. M., Breen, S. L., \& Godfrey, P. D. 2007, in IAU Symp. 242, Astrophysical Masers and Their Environments, ed. J. M. Chapman \& W. A. Baan (Cambridge: Cambridge Univ. Press), 213

Evans, N. J., II, Rawlings, J. M. C., Shirley, Y. L., \& Mundy, L. G. 2001, ApJ, 557,193

Glenn, J., et al. 2003, Proc. SPIE, 4855, 30

Goldreich, P., \& Kwan, J. 1974, ApJ, 189, 441

Haig, D. J., et al. 2004, Proc. SPIE, 5498, 78

Heiderman, A., et al. 2010, ApJ, 723, 1019

Hoare, M. G., Lumsden, S. L., Oudmaijer, R. D., Busfield, A. L., King, T. L., \& Moore, T. L. J. 2004, in ASP Conf. Ser. 317, Milky Way Surveys: The Structure and Evolution of Our Galaxy, ed. D. Clemens, R. Shah, \& T. Brainerd (San Francisco, CA: ASP), 156

Krumholz, M. R., \& McKee, C. F. 2008, Nature, 451, 1082

Kurtz, S., Cesaroni, R., Churchwell, E., Hofner, P., \& Walmsley, C. M. 2000, in Protostars and Planets IV, ed. V. Mannings, A. P. Boss, \& S. S. Russell (Tucson, AZ: Univ. Arizona Press), 299

Lada, C. J., Lombardi, M., \& Alves, J. F. 2010, ApJ, 724, 687

Ladjal, D., Justtanont, K., Groenewegen, M. A. T., Blommaert, J. A. D. L., Waelkens, C., \& Barlow, M. J. 2010, A\&A, 513, A53

Longmore, S. N., Burton, M. G., Barnes, P. J., Wong, T., Purcell, C. R., \& Ott, J. 2007, MNRAS, 379, 535
Matthews, H., et al. 2009, AJ, 138, 1380

McKee, C. F., \& Ostriker, E. C. 2007, ARA\&A, 45, 565

Mezger, P. G., \& Henderson, A. P. 1967, ApJ, 147, 471

Minier, V., Burton, M. G., Hill, T., Pestalozzi, M. R., Purcell, C. R., Garay, G., Walsh, A. J., \& Longmore, S. 2005, A\&A, 429, 945

Molinari, S., et al. 2010, PASP, 122, 314

Motte, F., Bontemps, S., Schilke, P., Schneider, N., Menten, K. M., \& Broguière, D. 2007, A\&A, 476, 1243

Motte, F., Schilke, P., \& Lis, D. C. 2003, ApJ, 582, 277

Mottram, J. C., Hoare, M. G., Lumsden, S. L., Oudmaijer, R. D., Urquhart, J. S., Sheret, T. L., Clarke, A. J., \& Allsopp, J. 2007, A\&A, 476, 1019

Ossenkopf, V., \& Henning, T. 1994, A\&A, 291, 943

Peretto, N., \& Fuller, G. A. 2009, A\&A, 505, 405

Peters, T., Banerjee, R., Klessen, R. S., Mac Low, M.-M., Galván-Madrid, R., \& Keto, E. R. 2010a, ApJ, 711, 1017

Peters, T., Mac Low, M.-M., Banerjee, R., Klessen, R. S., \& Dullemond, C. P. 2010b, ApJ, 719, 831

Price, S. D., Egan, M. P., Carey, S. J., Mizuno, D. R., \& Kuchar, T. A. 2001, AJ, 121,2819

Purcell, C. R., Hoare, M. G., \& Diamond, P. 2008, in ASP Conf. Ser. 387, Massive Star Formation: Observations Confront Theory, ed. H. Beuther, H. Linz, \& T. Henning (San Francisco, CA: ASP), 389

Purcell, C. R., Longmore, S. N., Burton, M. G., Walsh, A. J., Minier, V., Cunningham, M. R., \& Balasubramanyam, R. 2009, MNRAS, 394, 323

Rathborne, J. M., Jackson, J. M., Chambers, E. T., Simon, R., Shipman, R., \& Frieswijk, W. 2005, ApJ, 630, L181

Rathborne, J. M., Jackson, J. M., Chambers, E. T., Stojimirovic, I., Simon, R., Shipman, R., \& Frieswijk, W. 2010, ApJ, 715, 310

Rathborne, J. M., Jackson, J. M., \& Simon, R. 2006, ApJ, 641, 389

Rathborne, J. M., Jackson, J. M., Zhang, Q., \& Simon, R. 2008, ApJ, 689, 1141

Rathborne, J. M., Simon, R., \& Jackson, J. M. 2007, ApJ, 662, 1082

Reach, W. T., et al. 2006, AJ, 131, 1479

Reid, M. J., et al. 2009, ApJ, 700, 137

Robitaille, T. P., Whitney, B. A., Indebetouw, R., Wood, K., \& Denzmore, P. 2006, ApJS, 167, 256

Robitaille, T. P., et al. 2008, AJ, 136, 2413

Rosolowsky, E., et al. 2010, ApJS, 188, 123

Schlingman, W., et al. 2011, ApJ, submitted

Schuller, F., et al. 2009, A\&A, 504, 415

Shirley, Y. L., Evans, N. J., II, Young, K. E., Knez, C., \& Jaffe, D. T. 2003, ApJS, 149,375

Shu, F. H., Adams, F. C., \& Lizano, S. 1987, ARA\&A, 25, 23

Simon, R., Rathborne, J. M., Shah, R. Y., Jackson, J. M., \& Chambers, E. T. 2006, ApJ, 653, 1325

Smith, H. A., Hora, J. L., Marengo, M., \& Pipher, J. L. 2006, ApJ, 645, 1264

Soille, P. 1999, Morphological Image Analysis (Berlin: Springer)

Tielens, A. G. G. M. (ed.) 2005, in The Physics and Chemistry of the Interstellar Medium (Cambridge: Cambridge Univ. Press), 255

Urquhart, J. S., Busfield, A. L., Hoare, M. G., Lumsden, S. L., Clarke, A. J., Moore, T. J. T., Mottram, J. C., \& Oudmaijer, R. D. 2007a, A\&A, 461, 11

Urquhart, J. S., Hoare, M. G., Lumsden, S. L., Oudmaijer, R. D., \& Moore, T. J. T. 2008a, in ASP Conference Ser. 387, Massive Star Formation: Observations Confront Theory, ed. H. Beuther, H. Linz, \& T. Henning (San Francisco, CA: ASP), 381

Urquhart, J. S., et al. 2007b, A\&A, 474, 891

Urquhart, J. S., et al. 2008b, A\&A, 487, 253

Urquhart, J. S., et al. 2009a, A\&A, 501, 539

Urquhart, J. S., et al. 2009b, A\&A, 507, 795

Xu, Y., Reid, M. J., Menten, K. M., Brunthaler, A., Zheng, X. W., \& Moscadelli, L. 2009, ApJ, 693, 413

Zinnecker, H., \& Yorke, H. W. 2007, ARA\&A, 45, 481 